\title{
Reproductive health update trainings for health workers in North Eastern Province, Garissa
}

Kenya Ministry of Health

Population Council

Follow this and additional works at: https://knowledgecommons.popcouncil.org/departments_sbsr-rh

Part of the Gender and Sexuality Commons, International Public Health Commons, Obstetrics and Gynecology Commons, Public Health Education and Promotion Commons, and the Women's Health Commons How does access to this work benefit you? Let us know!

\section{Recommended Citation}

Kenya Ministry of Health and Population Council. 2006. "Reproductive health update trainings for health workers in North Eastern Province, Garissa." Nairobi: Ministry of Health and Population Council. 




\section{Reproductive Health Update Trainings for Health Workers in North Eastern Province, Garissa}

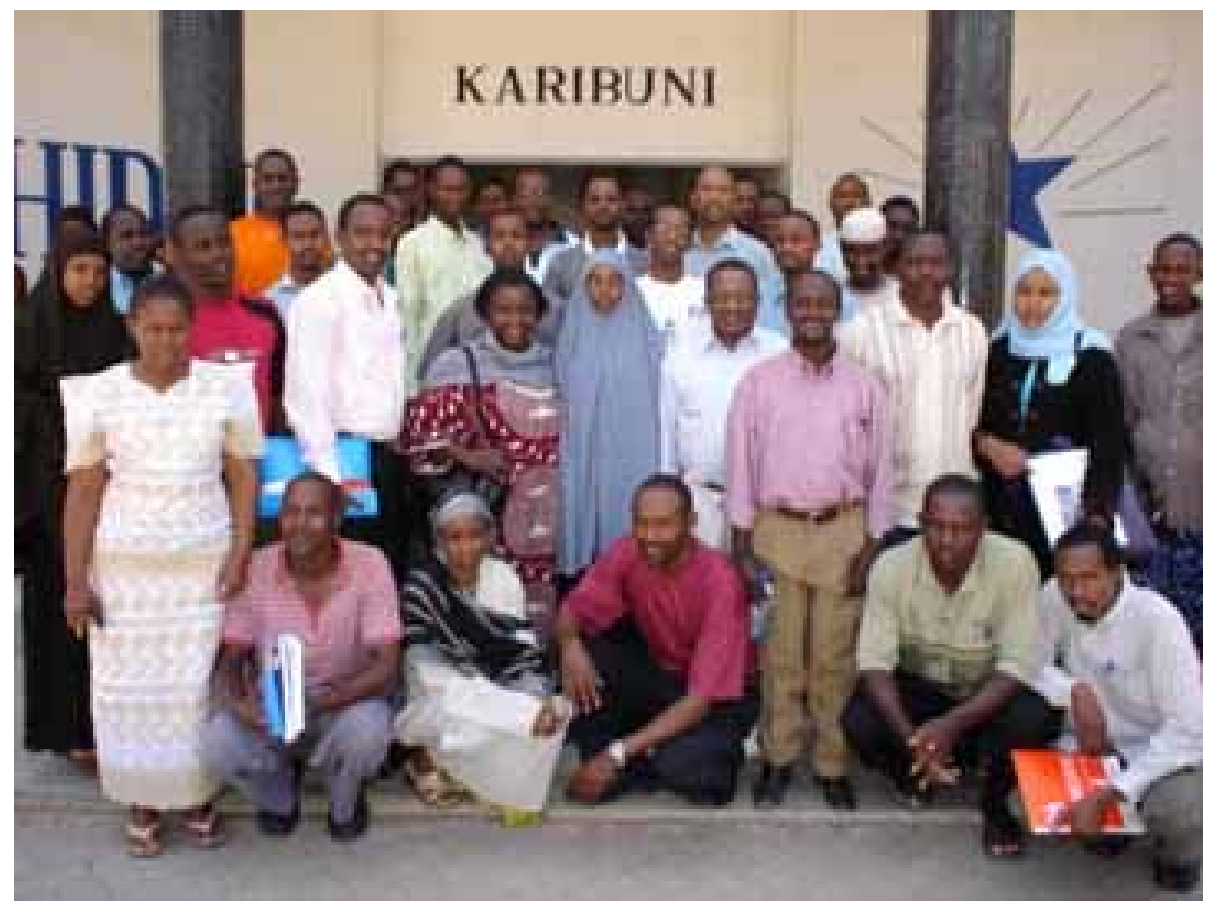

\section{Training Health Service Providers as Change Agents}

\section{6}

This study/publication is made possible by the generous support of the American people through the United States Agency for International Development (USAID) under the terms of Cooperative Agreement No. HRN-A-00-98-00012-00 and Population Council In-house project number 8016.53093. The contents are the responsibility of the FRONTIERS Program and do not necessarily reflect the views of USAID or the United States Government 


\section{ACKNOWLEDGEMENTS}

The training was organised by the population Council's Frontiers in Reproductive Health program (FRONTIERS), Nairobi office, through the Provincial Medical Officer's (PMO) office, North Eastern Province.

We would like to thank the various District Health Management Teams (DHMTs) in North Eastern Province for supporting the training and allowing their staff members to travel to Garissa for the training. We would like to thank the Head of Division of Reproductive Health, Ministry of Health Headquarters for sending their busy staff for the first two trainings where they presided over the opening and closing sessions together with the PMO besides giving various lectures on key management topics like supportive supervision and other educative sessions.

On behalf of the women and children of North Eastern Province who will benefit from the training we would like to thank very sincerely our partners UNICEF and DANIDA offices in Garissa, who supported the training financially and enabled us to reach the large number of health service providers. Special thanks go to the staff of UNICEF, Zeinab Ahmed and Hussein Golicha for their inspiring talks and experiences they shared with the participants. In the same way we would like to express our gratitude to the staff of DANIDA, Dr. Gunter., Dr. Chakava and Dr. Maurice for their support during all the training sessions.

We would also like to express our appreciation to management and staff of Garissa Provincial General Hospital for their cooperation and for allowing us to use their facility and patients during the practical sessions our training.

We acknowledge the contribution of Prof. Joseph Karanja of University of Nairobi's Department of Obstetrics and Gynaecology and Dr. Nancy Kidula, a practising Obstetrician Gynaecology in Nairobi for availing themselves and giving the participants up to date knowledge in reproductive health during the trainings.

This training would not have succeeded without the generous contribution of the people of United States of America through their organisation USAID who gave us the financial support for the program. 


\section{BACKGROUND TO THE TRAININGS}

The Somali community living in Kenya has practiced the severest form of FGM/C, infibulation, for centuries. To understand the context within which the practice takes place, and how its complications are managed, FRONTIERS undertook a diagnostic study in North Eastern Province (Wajir and Mandera districts) and in the Eastleigh area of Nairobi ${ }^{1}$. The diagnostic study found that the health system is ill equipped to serve women who have been cut, and particularly infibulated women who are pregnant and delivering. This stems from an overall weakness in the availability and quality of maternal and neonatal heath services in North Eastern Province.

Evidence from the Kenya Demographic and Health survey (2003) showed very low antenatal care attendance among this population, with about $70 \%$ not attending any ANC care, compared with less than $10 \%$ nationally. There is evidence in the literature that ANC attendance is associated with lower maternal morbidity and mortality as well as reduced perinatal mortality. This low attendance could be improved by community mobilisation and education through outreach activities by the health facility staff, during which the importance of early attendance for ANC, de-infibulation prior to delivery, and for organizing an attended delivery, preferably at a facility, are promoted. ANC consultations are also an opportunity to discourage mothers from cutting their daughters.

The health care providers are sometimes confronted with the life-threatening complications of FGM/C, mostly at the time following the cutting procedure as well as during delivery. The diagnostic study revealed that most of the health care providers were not confident in handling these complications. Infibulated pregnant women need a different management approach to pregnancy, delivery and the post-partum phase. High levels of complications are likely in any community practicing FGM/C, especially those where the most severe forms are common as seen among the Somali of North Eastern Province of Kenya. Health-care providers therefore required having the skills to treat and counsel these women. Unfortunately for these women, referral centres are often non-existent which means that, there is no access to the highly qualified services needed.

The health workers are part of the community themselves, such as fathers and mothers, uncles and aunts, play an important role in the decision as to whether or not to circumcise the female members of their family. Female health workers may be excised themselves. In addition, and especially in urban areas, health workers are increasingly being requested to perform infibulations and re-infibulations. In areas of high prevalence of type III FGM/C, women and their husbands often ask for re-infibulation

1 Jaldesa GW, Askew I, Njue C, Wanjiru M. Female genital cutting among the Somali of Kenya and management of its complications. Population Council: Nairobi, Kenya 
after the delivery of the baby. Many nurses are responding to these requests, with the justification that they can do it more safely than traditional practitioners, and because they can supplement their income, despite the fact that the practice is illegal and punishable. They should advise women and their husbands against re-infibulation after delivery and recommend that families do not seek FGM/C for their daughters. Health workers are well placed to counsel women suffering from psychological or sexual problems related to FGM/C. Again, however, they often lack the essential knowledge and skills needed to provide good counselling. As with other health-risk behaviours, health workers play an important role in any IEC and behaviour change campaign addressing the issue of FGM/C in communities.

Specific recommendations were made in the diagnostic study report of ways in which the health system could strengthen these services so as to competently manage women with FGM/C. Improved management of complications associated with FGM/C should be within the framework of improving safe motherhood services generally. Interventions with health providers should also contribute to abandonment efforts, through ensuring that staff adhere to $\mathrm{MOH}$ policy and become involved in communitylevel discussions to create a climate for behaviour change.

As a contribution to reducing maternal morbidity and mortality, these interventions will provide lessons that the $\mathrm{MOH}$ can use in its efforts to achieve the Millennium Development Goals, through demonstrating the feasibility and effect of strengthening maternal health services among this distinct population. In addition, it will identify the feasibility of working with communities that have proved highly resistant to external influences on traditional practices such as FGM/C. Links have been established with UNICEF, which with funding from the Italian Development Cooperation, is working in Garissa, Ijara and Moyale districts and nationally, to also address FGM/C. As far as possible, the interventions implemented through this project will be aligned with those of UNICEF so that common results can be found.

\section{OBJECTIVES}

\section{General Objective}

To reduce the suffering caused by FGM/C among the Somali community in Kenya through improving the health system's capacity to manage women who have undergone genital cutting, and through encouraging the community's abandonment of the practice. 


\section{Specific Objectives}

To develop a training curriculum and materials to improve management of pregnancy, delivery and postpartum care for women with genital cutting, to manage gynaecological and sexual complications among women who have been cut, and to advocate against the practice, that can be used for pre- and in-service training by health workers ${ }^{2}$ providing antenatal and basic obstetric care.

To strengthen the capacity of Provincial and District Health Management Teams in North Eastern Province to supervise and support the provision of antenatal, labour, delivery, postnatal and newborn care services for women (and their newborn) who have undergone FGM/C.

To improve the quality of care received by women seeking antenatal, obstetric and gynaecological services in North Eastern Province through strengthening the capacity of clinics and clinic staff to provide these services among a population that practises FGM/C.

\section{DISCUSSIONS AND TRAINING ACTIVITIES}

\section{The Training at Garissa Provincial General Hospital}

The training was conducted in seven-day sessions. There were five training sessions conducted with a total of 145 health workers from various cadres, facilities and districts. Each training session had theoretical sessions where the participants updated their knowledge on common conditions that contribute to maternal mortality and morbidity in their work environment.

After the cross-cutting issues that contributed to poor service utilization by the patients such as infection prevention, data collection and utilization, emergency preparedness, communication and counseling techniques and introduction to FGM/C as a traditional practice, which is universal in the project area, the participants toured the Hospital which is a center of excellence and referral center for the region. The aim of this guided orientation tour is to have a quick appraisal of reproductive health services and the organization of the provincial hospital including its management and comparing it to their place of work. Each training session was concluded with a selected video show, which was relevant to the preceding session.

Recognizing that there is a limited medical literature for the participants and to build their level of knowledge that they can take back to their institutions and share with their colleagues, each participants was issued with a well selected resource materials.

2 The curriculum is intended for the following cadres of health workers: nurses, nurse-midwives, clinical officers, medical officers, and doctors. 
The materials used for the trainings included the following;

- WHO Trainee's manual on Female Genital Mutilation,

- Integrating the Prevention and the Management of the Health Complications into the curricula of nursing and midwifery,

- The Population Council's / MOH/UON Essential Obstetric Care Manual for Health Service Providers in Kenya,

- Management of Complications, Pregnancy, childbirth and the postpartum period in the presence of Female Genital Cutting / Mutilation, Manual for health workers,

- Female Genital Cutting among the Somali of Kenya and Management of its Complications, a report for Population Council, Kenya office, among many other materials.

\section{The Training ORganization}

The first training constituted of five District Health Management Team (DHMT) members from all the five participating districts and five members of Provincial Health Management Team (PHMT). From this team one member was chosen from each district to be trained as a facilitator for future trainings. This individual was also the one coordinating the trainings in their respective districts. All the subsequent trainings were co-presented with facilitators from Nairobi and the ones from the region. The aim of this was to develop the manpower from the region so that they can conduct their own trainings in the field of reproductive health especially FGM/C after the end of the program life. They were also empowered to give supportive supervision in their respective districts.

The training of health service providers from North Eastern recognised that the region has several health problems. These areas were identified and the participants given both theoretical and practical trainings. The key areas covered are antenatal care, care of women during labour, delivery and postpartum period as well as the care of newborn. Other common medical conditions that occur in pregnancy observed in North Eastern Province such as malaria, anaemia and hypertensive diseases in pregnancy were also taught.

The training team recognised that the prevalence of $\mathbf{H I V}$ in the region is about $3.5 \%$ as per hospital reports. Though the prevalence of HIV in this region is low, the health care providers must realize the importance of HIV infection and the need for action. Good counselling skills are important to combat the psychological aspects of HIV infection in pregnancy. Through this training the participants were given updates on the national HIV trends and taught on the care of HIV in pregnancy including prevention of mother 
to child transmission, which is incorporated into the antenatal care. Special attention is given to nutrition, risk reduction behaviour, counselling on infant feeding and family planning services. Changes in midwifery and obstetric practice are required due to HIV infection in pregnancy.

Starting day four, the participants were divided into groups whereby they went through a hands-on practical session in labour ward and the antenatal clinics including family planning and voluntary counseling and testing for HIV units. While in these units the participants took part in care, counseling as well as health education talks on various topics including FGM/C. Those in the labour ward were given the practice of monitoring women in labour, and depending on the FGM/C status of the patient, the participants practised de-infibulation and counselled against re-infibulation. (See the attached appendix for the tasks given to participants to perform at various departments).

Considering that there is a strong attachment of religion as a factor for performing FGM/C in this community, a belief strongly held by the health workers from the community, all the training sessions committed some time for an Islamic scholar to talk on the role of FGM/C in Islam. This was aimed at de-linking Islam and FGM/C. The participants also during all the trainings watched two video shows on FGM/C.

One of the films was a 16-minutes production by UNICEF, Women on the run, featuring one prominent Kenyan woman who escaped FGM/C and thus successfully completed her education and got married against the myths held by her community and developed to become a cabinet minister in Kenya. Also with her was a world marathon runner who also escaped the practice and succeeded in her community. The video ended with a success story of a traditional practitioner giving up the practice on recognizing the problems associated with the practice. The other video was a $\mathrm{WHO}$ documentary: The road to change, which retraces the history of FGM/C by placing it in the perspective of other traditional practices that have affected women's health and sexuality across civilizations and throughout history. It also showed the grassroots work undertaken by NGOs and activists to increase communities' awareness about FGM/C, as well as the action undertaken by the National Committee Against FGM/C in Burkina Faso including alternative rites of passage to bring an end to the practice. It was emphasized in the video the fact that FGM/C is not a religious practice and it is an act against humanity.

At the end of the seven-day training session, each participant wrote down in duplicates, the activities they hope to carry out at their workstation on return. The facilitators from Population Council retained a copy of this individual action while the participant retained their copy. The purpose of this is to keep the fire of this training to continue burning and it is also a pledge from the participants to make changes at their facilities so as to improve the quality of service to their clients. The DHMT, the Council and other donors will use the copy retained by the Council facilitators during supportive supervision and follow up visits. 
At the beginning and the end of every training session the participants undertook a written test (pretest and posttest). The purpose of these tests was to understand the areas of their weakness that required emphasis during the training and to indirectly assess the quality of the training. The posttest also examined the quality of knowledge gained which may reflect on their future practice.

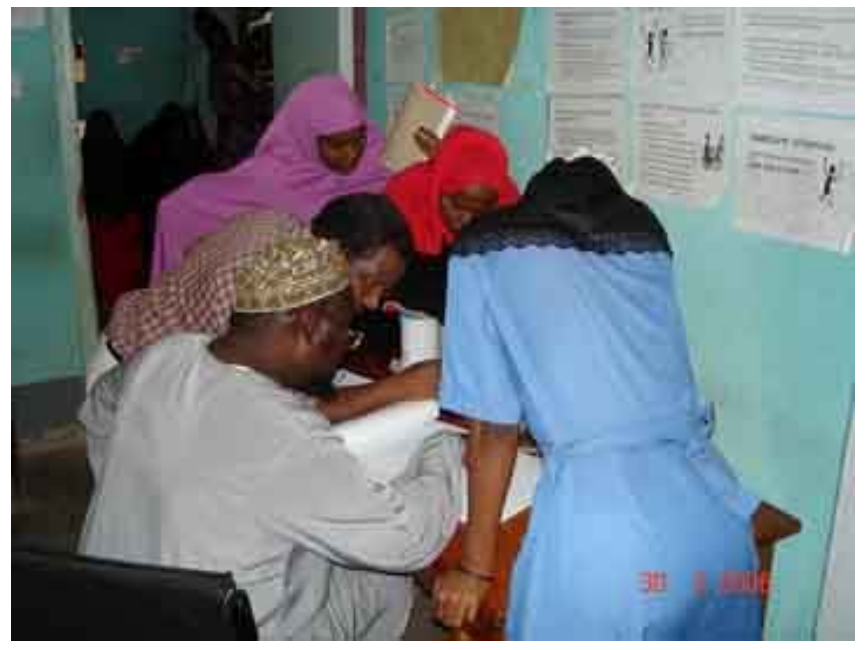

Facilitators demonstrating how to fill antenatal register to the participants at the ANC clinic. Other than the training participants, the hospital staff working in various departments including the medical training students benefited as they were taught along with the participants.

Through this we hope to build the capacity of Garissa PGH and it's staff to do proper documentation. The participants were provided with various registers provided by DANIDA programme aimed at strengthening the information collection.

A local facilitator giving lectures in the classroom. One component of the training is to develop the capacity of health workers in the region to be trainers in reproductive health and be part of the advocacy teams for women rights.

The 'local' trainers were trained through mentorship and currently can conduct their own training with minimal support from outside.

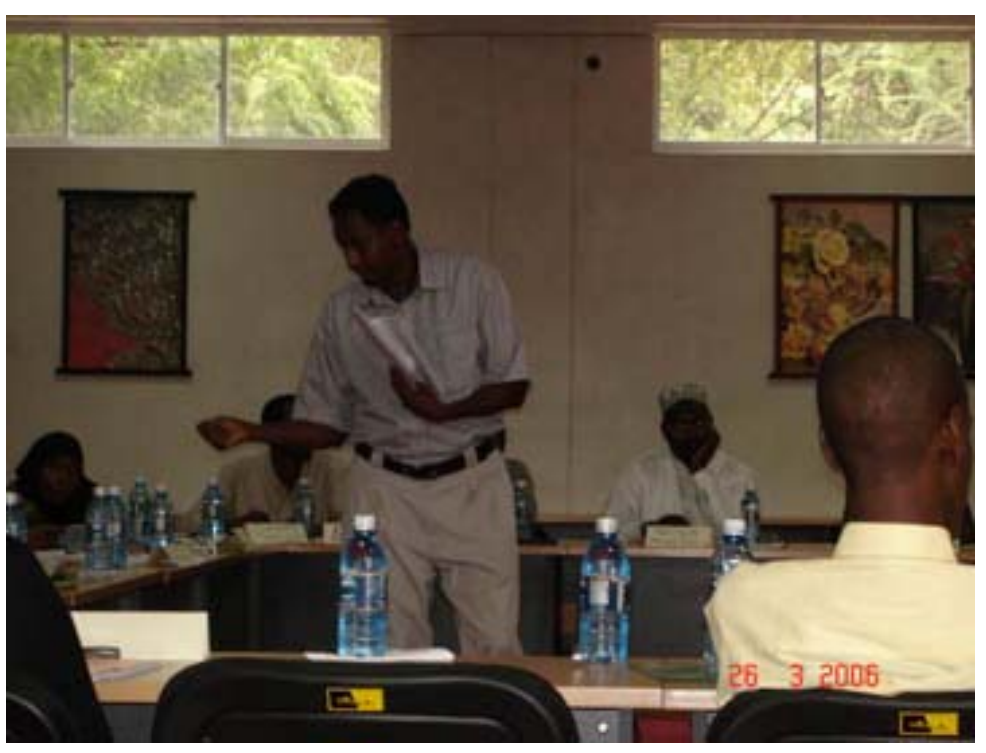




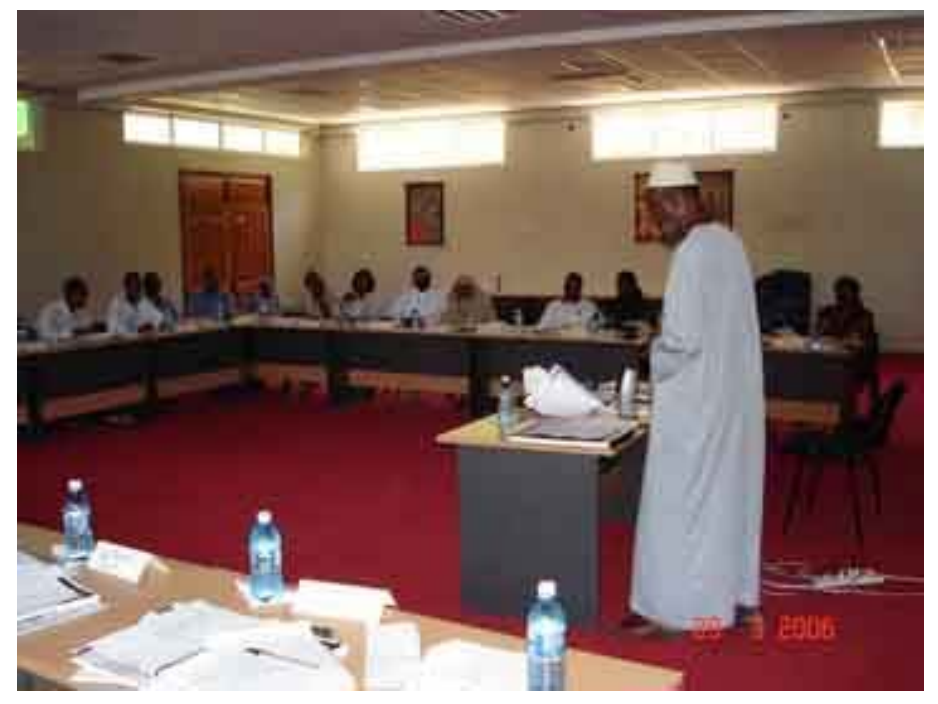

A religious leader giving lectures on FGM/C and Islam demystifying the wrong perspective that FGM/C act required by Islam. At the end of every training the participants were asked to declare whether they think FGM/C is a practice required by Islam and how many of them are willing to talk against the practice with some confidence.

This session is usually quite interactive where the participants discuss around all the myths, rumours and all the misconceptions they have had concerning FGM/C and Islam.

During one of the training sessions, His Excellency the Ambassador of the Royal Danish Embassy in Nairobi visited our training session which was co-sponsored by DANIDA and UNICEF offices in Garissa.

Accompanying the ambassador were the Provincial commissioner, North Eastern Province and the District Commissioner Garissa among other prominent guests.
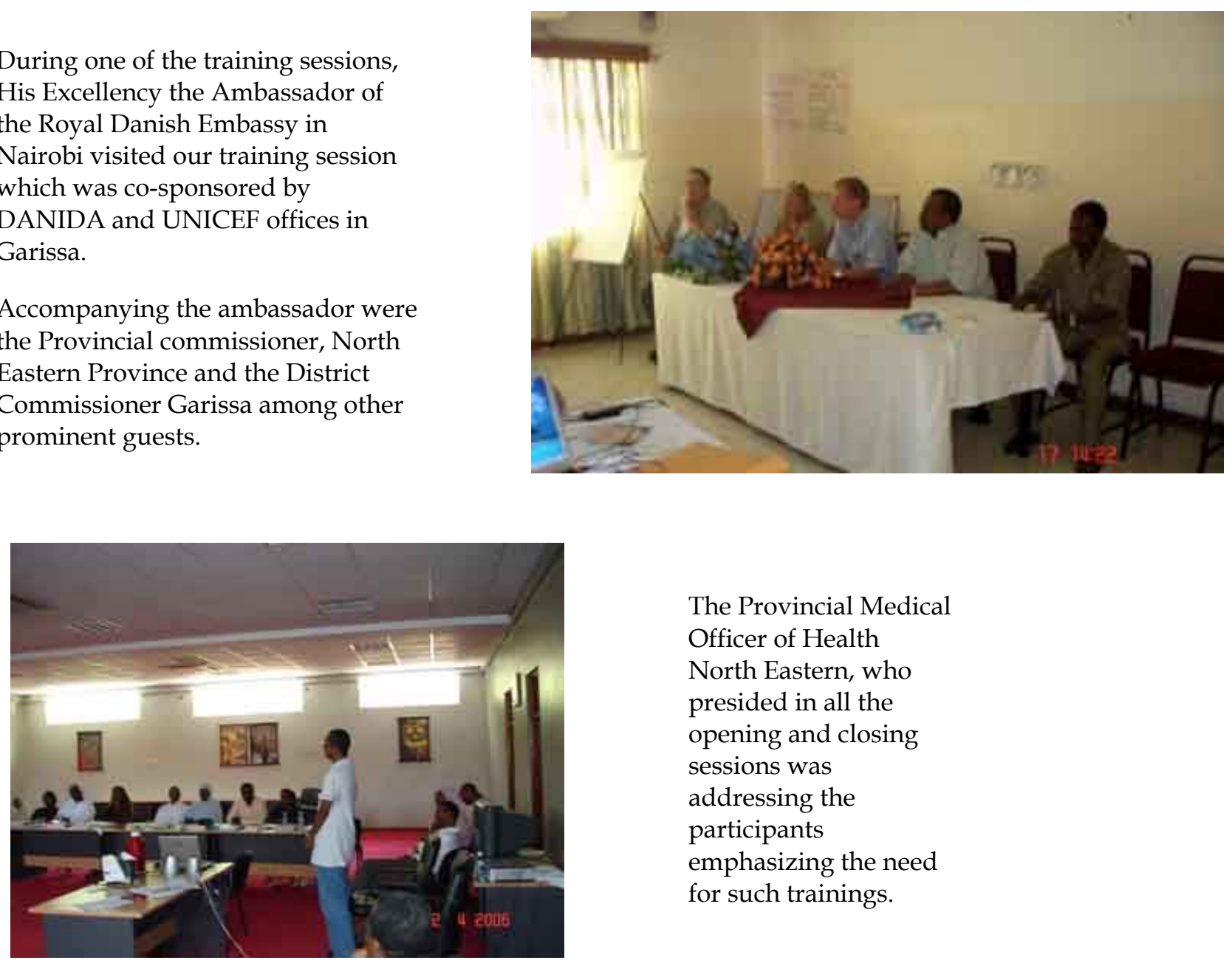

The Provincial Medical

Officer of Health

North Eastern, who

presided in all the

opening and closing

sessions was

addressing the

participants

emphasizing the need

for such trainings. 


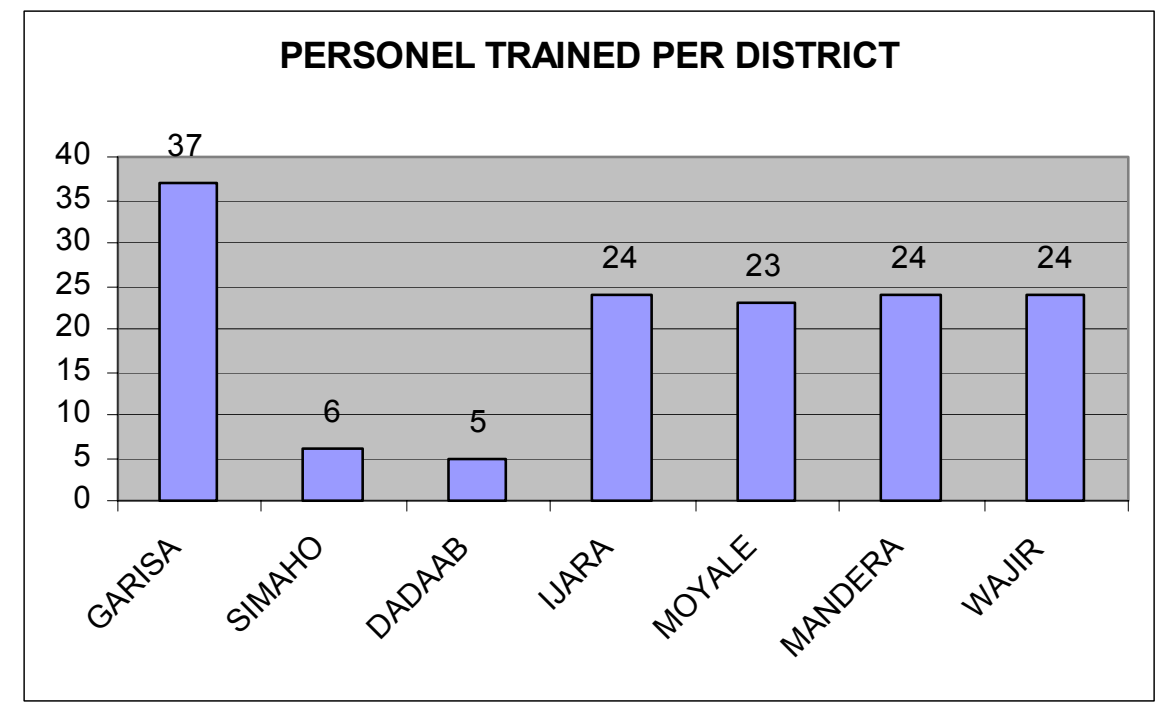

Garissa distict took advantage of the home ground and sent in the highest number of trainees. Other major beneficiaries included a private nursing home SIMAHO and the Dadaab-GTZ managed refugee health hospitals that sent five participants. All the other districts sent in almost equal number of participants.



The nurses were the majority of all the health workers. The others who were trained included Medical Officers, public health officers, health information officers and hospital administrators. The Nurses are the bulk of health service providers and it was our intention to train as many as we could. 


\section{Performance of the participants from different districts}

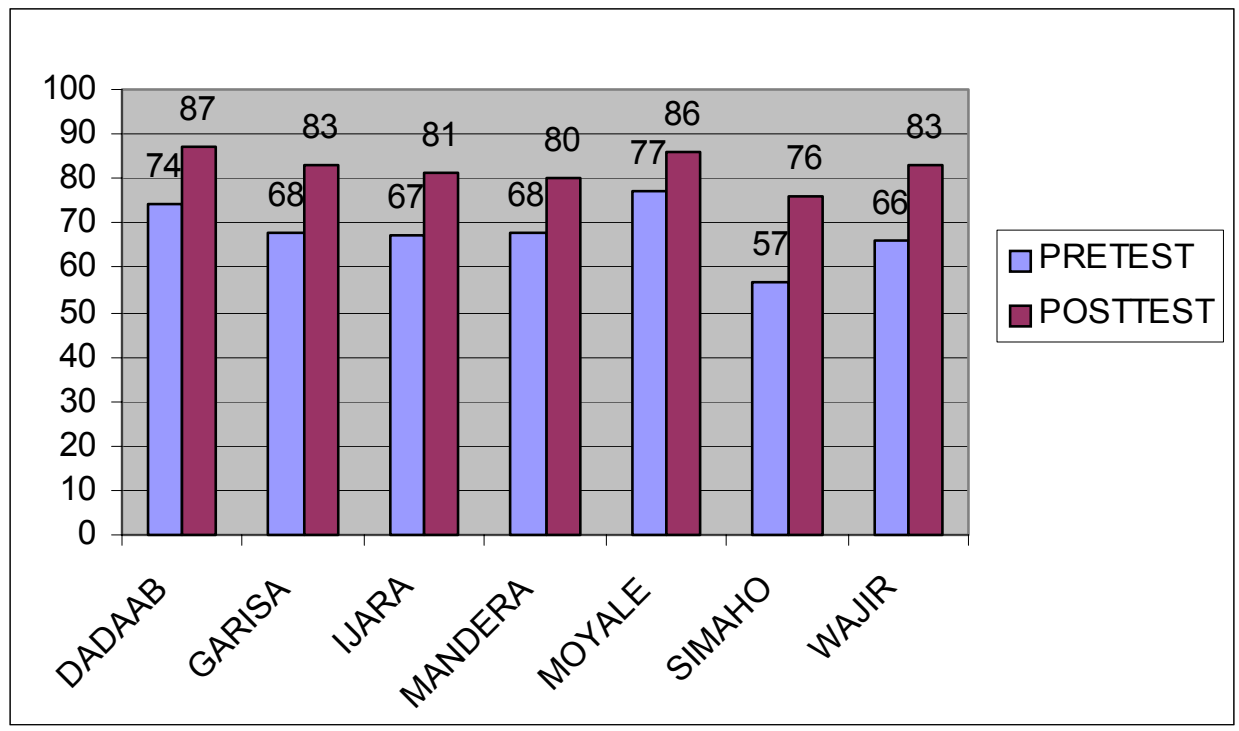

All the districts recorded improved performance at the posttest as compared to the pretest. The participants from SIMAHO and Wajir recorded the best improvement in their posttest performance.

\section{Evaluation of the training by participants}

At the end of every training session the participants were asked to evaluate the training they had undertaken over the previous seven days. Below are summary of their views.

1. Facilitators gave a recommendable job.

2. Gained more knowledge on $\mathrm{RH}$ in the training.

3. It was very good. Continue with the same spirit.

4. Scope of health workers are captured and sensitised.

5. Course was excellent and participation of participants was good.

6. Very educative course. Improves the lives of the family pillars i.e. mothers and child especially the girl child. Bravo trainers!! You have made it and you are very experienced facilitators.

7. Future training should be conducted at grass root level so that other health service providers in cadres not included in these trainings can benefit.

8. The training has been standardized. Knowledge and skills was gained. The videotapes and hospital visits have added more to the training.

9. Training was educative and timely as FGM/C is chronic and Somali girls are dying daily due to complications related to FGM/C. 
10. Absolutely necessary for the health care managers and care providers

11. The course, timing and content were relevant to the participants

12. The timing was well planned, prepared and conducted by professionals

13. Issues of FGM/C especially the Islamic perspective has come out crystal clear

14. Training was very educative

15. Course was good, I gained a lot of knowledge

16. Course excellent, Educative and refreshing.

17. Congested time-table. There is a need to add more time for participants to digest and revise. Maybe extend the training by two more days

18. I Congratulate the organizers for bringing learned friends especially experienced practising doctors and lecturers from the university.

19. The facilitators were friendly always.

20. Thank the managers and organizers of the course.

\section{Recommendations on future trainings by participants}

The participants were asked to suggest ways of improving the future training based on their experience. These are the summary of their recommendations.

1. To make the training more practical, it should be integrated involving religious leaders, health workers and other stakeholders.

2. Incorporate theory with practical sessions in hospital and those mothers who do the FGM/C - about three days practical.

3. Increase the duration of the training to 2 weeks to give more practical sessions.

4. A similar course should be done for all health workers especially those from relevant departments.

5. The course should be done at district level so as to give opportunities to those who cannot travel outside their facilities.

6. Involve community leaders on sensitization, as they are the agents of change in the society.

7. Follow up to the districts should be done for monitoring and evaluation after these courses are done to ensure service providers put what they have learnt into practice.

8. Please issue certificates of participation, as it is very important to us.

9. The venue is conducive for the training so please keep this as the place for future trainings. 


\section{CONCLUSions}

- There is need for follow up of the participants to ensure that they will practice what they learnt and implement their individual action plans.

- There is need for extended practical trainings session for those from busy health facilities to give them more hands on experience.

The following were recruited as facilitators for various workshops.

- Dr. Ahmedin H. Omar - Wajir District - 0721667326, Deen_Omar@yahoo.com

- Mohamed Salat Dagane - Garissa District/PHMT 0721424557, salat_2004@yahoo.com

- Mohamed Hambulle - Moyale District 0720314851 or 0734348417

- Adam M. Mohamed-Mandera District 0722586177, adamqone@hotmail.com

- Siyat Moge - Ijara District 0721298302, siyatgure@yahoo.ac.uk 


\section{APPENDICES}

\section{Appendix 1. Action plans}

Below, is a selection of individual and district workplans produced by the participants during the trainings.

\section{MANDERA DiSTRICT}

\begin{tabular}{|l|l|l|l|l|}
\hline \multicolumn{5}{|c|}{ Adam Mohamed, DPHO, Mandera District Hospital } \\
\hline ACTIVITY & WHERE & TIME & RESPONSIBILITY & RESOURCES \\
\hline $\begin{array}{l}\text { Sensitize PH staff on RH, } \\
\begin{array}{l}\text { FGM/C, child law, human } \\
\text { right and their role }\end{array}\end{array}$ & $\begin{array}{l}\text { Health } \\
\text { office }\end{array}$ & $24 / 12 / 05$ & Adam & Sodas and notebooks \\
\hline $\begin{array}{l}\text { Supervise infection control } \\
\text { team fortnight }\end{array}$ & MDH & $\begin{array}{l}\text { From 23rd } \\
\text { Dec 05 }\end{array}$ & Adam & Disinfectants/Packets \\
\hline $\begin{array}{l}\text { Initiate and carry out RH } \\
\text { school health } \\
\text { program/institution }\end{array}$ & Institutions & $\begin{array}{l}\text { From } \\
8 / 02 / 06\end{array}$ & Adam & Transport \\
\hline
\end{tabular}

\begin{tabular}{|l|l|l|l|l|}
\hline \multicolumn{5}{|c|}{ Suleiman H. Omar, Physiotherapist, Mandera District Hospital } \\
\hline ACTIVITY & WHERE & TIME & RESPONSIBILITY & RESOURCES \\
\hline $\begin{array}{l}\text { Advice mothers who come with } \\
\text { delayed milestone children to } \\
\text { deliver at hospitals }\end{array}$ & Physio dept & $19 / 12 / 05$ & Self & None \\
\hline $\begin{array}{l}\text { Educate and sensitize mothers on } \\
\text { adverse effect of FGM/C }\end{array}$ & Physio dept & $16 / 1 / 06$ & Self & None \\
\hline $\begin{array}{l}\text { Visit ANC clinic and teach pregnant } \\
\text { mothers on pelvic floor MMS } \\
\text { exercises (group exercises) }\end{array}$ & ANC clinic & $24 / 2 / 06$ & Self & None \\
\hline $\begin{array}{l}\text { Start and stress infection prevention } \\
\text { techniques in handling patients and } \\
\text { protecting staff }\end{array}$ & $\begin{array}{l}\text { Physio dept } \\
\text { and the } \\
\text { wards }\end{array}$ & $\begin{array}{l}\text { As soon as } \\
\text { I go back }\end{array}$ & Self and other staff & None \\
\hline
\end{tabular}

\begin{tabular}{|l|l|l|l|l|}
\hline \multicolumn{5}{|c|}{ Issack Maalim Eliyas, Pharm Tech, Mandera District Hospital } \\
\hline ACTIVITY & WHERE & TIME & RESPONSIBILITY & RESOURCES \\
\hline $\begin{array}{l}\text { Dissemination of RH } \\
\text { programmes to pharmacy staff }\end{array}$ & $\begin{array}{l}\text { Madera District } \\
\text { Hospital }\end{array}$ & $23 / 12 / 05$ & Self & None \\
\hline Promote infection prevention & $\begin{array}{l}\text { MDH pharmacy } \\
\text { dept }\end{array}$ & $\begin{array}{l}\text { From Jan } \\
2006\end{array}$ & Self/Staff & None \\
\hline Ensure rational use of drugs & MDH & $\begin{array}{l}\text { From Jan } \\
2006\end{array}$ & Self & None \\
\hline $\begin{array}{l}\text { Ensure supportive } \\
\text { pharmaceutical supervision }\end{array}$ & MDH & $\begin{array}{l}\text { From Jan } \\
2006\end{array}$ & Self & None \\
\hline
\end{tabular}




\begin{tabular}{|l|l|l|l|l|}
\hline \multicolumn{4}{|c|}{ Derrow Maalim Gamow, Health Education Officer, Mandera District Hospital } \\
\hline ACTIVITY & WHERE & TIME & RESPONSIBILITY & RESOURCES \\
\hline $\begin{array}{l}\text { Give feedback to health } \\
\text { education staff }\end{array}$ & MDH & $19 / 12 / 05$ & Derrow & None \\
\hline $\begin{array}{l}\text { Initiate continuous medical } \\
\text { education committee }\end{array}$ & MDH & $6 / 1 / 06$ & Derrow & None \\
\hline $\begin{array}{l}\text { Strengthen health programme } \\
\text { in ANC (health talk timetable) }\end{array}$ & MDH & $10 / 1 / 06$ & Derrow & None \\
\hline $\begin{array}{l}\text { Organise one day meeting with } \\
\text { TBA's }\end{array}$ & $\begin{array}{l}\text { Central } \\
\text { Divisions }\end{array}$ & $24 / 1 / 06$ & Derrow & None \\
\hline
\end{tabular}

\begin{tabular}{|l|l|l|l|l|}
\hline \multicolumn{5}{|c|}{ Omondi Gogo, KRCHN, Mandera District Hospital } \\
\hline ACTIVITY & WHERE & TIME & RESPONSIBILITY & RESOURCES \\
\hline Set an emergency tray & $\begin{array}{l}\text { ANC } \\
\text { Maternity }\end{array}$ & $14 / 12 / 05$ & $\begin{array}{l}\text { In charge of Maternity and } \\
\text { ANC }\end{array}$ & $\begin{array}{l}\text { Trays and emergency } \\
\text { drugs and equipment }\end{array}$ \\
\hline $\begin{array}{l}\text { Strengthen infection } \\
\text { prevention method }\end{array}$ & $\begin{array}{l}\text { All wards in } \\
\text { MDH }\end{array}$ & $14 / 12 / 05$ & In charge of all wards & None \\
\hline $\begin{array}{l}\text { Plot ante-natal delivery } \\
\text { monitoring chart }\end{array}$ & $\begin{array}{l}\text { Ante-natal RM } \\
\text { and maternity }\end{array}$ & $30 / 12 / 05$ & $\begin{array}{l}\text { In charge of MCH/FP and } \\
\text { maternity }\end{array}$ & Monitoring charts \\
\hline $\begin{array}{l}\text { Make sure all wards } \\
\text { have duty roster }\end{array}$ & All wards & $14 / 12 / 05$ & In charge of all wards & Plain papers \\
\hline $\begin{array}{l}\text { Feedback of the } \\
\text { seminar }\end{array}$ & $\begin{array}{l}\text { To all nursing } \\
\text { staff }\end{array}$ & $14 / 12 / 05$ & Omondi & Nil \\
\hline
\end{tabular}

\section{WAJIR DISTRICT}

\begin{tabular}{|l|l|l|l|l|}
\hline \multicolumn{5}{|c|}{ Dr. Ahmedin H. Omar, DMOH, Wajir District } \\
\hline ACTIVITY & WHERE & TIME & RESPONSIBILITY & RESOURCES \\
\hline $\begin{array}{l}\text { Establish an active } \\
\text { maternal mortality } \\
\text { review }\end{array}$ & DH/Sub DH & $\begin{array}{l}\text { January } \\
06\end{array}$ & $\begin{array}{l}\text { DMOH/ Incharge sub } \\
\text { District hospitals }\end{array}$ & None \\
\hline $\begin{array}{l}\text { Establish routine health } \\
\text { education for mothers in } \\
\text { MCH }\end{array}$ & $\begin{array}{l}\text { Wajir District } \\
\text { Hospital }\end{array}$ & $19 / 12 / 06$ & In charge MCH & $\begin{array}{l}\text { IEC materials on } \\
\text { reproductive } \\
\text { health }\end{array}$ \\
\hline $\begin{array}{l}\text { Provide supportive } \\
\text { supervision to all } \\
\text { facilities }\end{array}$ & Hospital/S.D. H & $19 / 12 / 05$ & DMOH & $\begin{array}{l}\text { Logistics and } \\
\text { personnel } \\
\text { allowance }\end{array}$ \\
\hline $\begin{array}{l}\text { Establish emergency } \\
\text { preparedness }\end{array}$ & $\begin{array}{l}\text { Health Centres, } \\
\text { Dispensaries }\end{array}$ & $\begin{array}{l}\text { Feb - } \\
\text { March 06 }\end{array}$ & $\begin{array}{l}\text { Nursing Officer in } \\
\text { charge and DPHN }\end{array}$ & $\begin{array}{l}\text { Supplies and } \\
\text { drugs }\end{array}$ \\
\hline $\begin{array}{l}\text { Sensitize communities } \\
\text { on FGM/C }\end{array}$ & $\begin{array}{l}\text { Wajir hospital, } \\
\text { Habasweni, Griftu } \\
\text { and Bute }\end{array}$ & $\begin{array}{l}\text { Feb - } \\
\text { March 06 }\end{array}$ & $\begin{array}{l}\text { DMOH } \\
\text { Ksh. 300,000 }\end{array}$ \\
\hline
\end{tabular}




\begin{tabular}{|l|l|l|l|l|}
\hline \multicolumn{3}{|c|}{ Mohamed Hussein Ali, Nursing Officer in Charge, Wajir District Hospital } \\
\hline ACTIVITY & WHERE & TIME & RESPONSIBILITY & RESOURCES \\
\hline Feedback to HMT & Hospital & $14 / 12 / 05$ & Self & $\begin{array}{l}\text { Space, staff and } \\
\text { time }\end{array}$ \\
\hline $\begin{array}{l}\text { Meeting with ward } \\
\text { incharges }\end{array}$ & Office & $18 / 12 / 05$ & Self & Office space \\
\hline $\begin{array}{l}\text { Strengthen infection } \\
\text { prevention in all depts. } \\
\text { Within the hospital }\end{array}$ & Hospital & Jan - Feb 06 & Self & $\begin{array}{l}\text { Supplies, } \\
\text { protocols and } \\
\text { guidelines }\end{array}$ \\
\hline $\begin{array}{l}\text { Strengthen FANC and } \\
\text { PMTCT in MCH and } \\
\text { maternity }\end{array}$ & MCH/Maternity & From 19/12/05 & Self & Registers \\
\hline $\begin{array}{l}\text { Follow up health } \\
\text { education activities }\end{array}$ & $\begin{array}{l}\text { MCH/Maternity } \\
\text { /VCT/OPD }\end{array}$ & From 19/12/05 & Self & materials on RH \\
\hline $\begin{array}{l}\text { Start and strengthen } \\
\text { MDR }\end{array}$ & Maternity & January 2006 & Self & $\begin{array}{l}\text { Discuss in HMT } \\
\text { meetings }\end{array}$ \\
\hline Start IP committees & Hospital & January 2006 & Self & Avail Jik, buckets \\
\hline Supervision & All departments & 2 weekly & Self & $\begin{array}{l}\text { Time, checklist, } \\
\text { Team }\end{array}$ \\
\hline
\end{tabular}

\begin{tabular}{|l|l|l|l|l|}
\hline \multicolumn{5}{|c|}{ Abdirahman Sheikh, Lab Technician, Wajir District Hospital } \\
\hline ACTIVITY & WHERE & TIME & RESPONSIBILITY & RESOURCES \\
\hline $\begin{array}{l}\text { Feedback on reproductive } \\
\text { health and lab staff }\end{array}$ & $\begin{array}{l}\text { Wajir District } \\
\text { Hospital }\end{array}$ & $22 / 12 / 05$ & Self & None \\
\hline $\begin{array}{l}\text { Start an infection control team } \\
\text { at laboratory }\end{array}$ & WDH & $23 / 12 / 05$ & Self & None \\
\hline $\begin{array}{l}\text { Ensure supportive supervision } \\
\text { and feedback is given }\end{array}$ & WDH & $2 / 2 / 06$ & Self & None \\
\hline $\begin{array}{l}\text { Ensure all ANC profile text is } \\
\text { done on time and proper } \\
\text { recording at registry }\end{array}$ & WDH & $19 / 12 / 05$ & Self & None \\
\hline
\end{tabular}

\begin{tabular}{|l|l|l|l|l|}
\hline \multicolumn{4}{|c|}{ Dr. Ahmedin Hassan Omar, DHMT, Wajir District Hospital } \\
\hline ACTIVITY & WHERE & TIME & RESPONSIBILITY & RESOURCES \\
\hline $\begin{array}{l}\text { Supportive supervision } \\
\text { for all facilities in the } \\
\text { district with emphasis on } \\
\text { staff members }\end{array}$ & Whole district & Quarterly & Dr. Ahmedin & $\begin{array}{l}\text { Logistic and } \\
\text { personnel } \\
\text { allowance }\end{array}$ \\
\hline $\begin{array}{l}\text { Feedback to DHMT on } \\
\text { staff members }\end{array}$ & $\begin{array}{l}\text { Wajir District } \\
\text { Hospital }\end{array}$ & $19-26.12 .06$ & Dr. Ahmedin & None \\
\hline $\begin{array}{l}\text { OJT to maternity staff on } \\
\text { partograph usage }\end{array}$ & D.H, SDH & January 2006 & Dr. Ahmedin & $\begin{array}{l}\text { Stationery for } \\
\text { partograph }\end{array}$ \\
\hline On MgS04 usage & DH, SDH & January 2006 & Dr. Ahmedin & $\begin{array}{l}\text { MgS04 } \\
\text { availability }\end{array}$ \\
\hline
\end{tabular}




\begin{tabular}{|l|l|l|l|l|}
\hline \multicolumn{5}{|c}{ Adam Abdirahman, Nurse, Eldas Dispensary } \\
\hline ACTIVITY & WHERE & TIME & RESPONSIBILITY & RESOURCES \\
\hline $\begin{array}{l}\text { Introduce duty } \\
\text { roster }\end{array}$ & Eldas dispensary & Immediately & In charge & Stationery \\
\hline $\begin{array}{l}\text { Introduction to } \\
\text { infection } \\
\text { prevention }\end{array}$ & Eldas dispensary & Immediately & In charge & $\begin{array}{l}\text { Posters, Manilla } \\
\text { paper }\end{array}$ \\
\hline $\begin{array}{l}\text { Implement } \\
\text { records and } \\
\text { registers }\end{array}$ & Eldas dispensary & Immediately & In charge & Jik, Water \\
\hline $\begin{array}{l}\text { Feedback to } \\
\text { community }\end{array}$ & $\begin{array}{l}\text { Colleagues and } \\
\text { community living } \\
\text { near the dispensary }\end{array}$ & Immediately & Nursing officer in charge & None \\
\hline $\begin{array}{l}\text { Create } \\
\text { awareness on } \\
\text { FGM/C }\end{array}$ & Eldas dispensary & Immediately & In charge & Posters \\
\hline ANC, Early visit & Eldas dispensary & Immediately & In charge & Transport \\
\hline $\begin{array}{l}\text { Set emergency } \\
\text { tray }\end{array}$ & Eldas dispensary & Immediately & In charge & $\begin{array}{l}\text { Tray and } \\
\text { emergency } \\
\text { equipment }\end{array}$ \\
\hline
\end{tabular}

\begin{tabular}{|l|l|l|l|l|}
\hline \multicolumn{5}{|c|}{ Abdi Adow Aden, KECHN, Wajir District Hospital } \\
\hline ACTIVITY & WHERE & TIME & RESPONSIBILITY & $\begin{array}{l}\text { RESOURCE } \\
\text { REQUIRED }\end{array}$ \\
\hline Feedback to M.C.N. & M.C.H staff & 15.12 .05 & Abdi & None \\
\hline Update emergency tray & $\begin{array}{l}\text { Maternity, } \\
\text { MCH }\end{array}$ & 30.12 .05 & Abdi & None \\
\hline $\begin{array}{l}\text { Strengthen infection } \\
\text { prevention }\end{array}$ & $\begin{array}{l}\text { M.C.F and } \\
\text { maternity }\end{array}$ & 7.01 .06 & Abdi & None \\
\hline $\begin{array}{l}\text { Strengthen medication in } \\
\text { MCH and Maternity }\end{array}$ & $\begin{array}{l}\text { MCH, } \\
\text { Maternity }\end{array}$ & 15.01 .06 & Abdi & None \\
\hline $\begin{array}{l}\text { Share update on RH (eg } \\
\text { Medical eligibility criteria) }\end{array}$ & MCH & 30.01 .06 & Abdi & None \\
\hline
\end{tabular}

\begin{tabular}{|l|l|l|l|l|}
\hline \multicolumn{5}{|c|}{ Soransora, Nursing Officer, Moyale District Hospital } \\
\hline ACTIVITY & WHERE & TIME & RESPONSIBILITY & $\begin{array}{l}\text { RESOURCE } \\
\text { REQUIRED }\end{array}$ \\
\hline Workshop Feedback & Hospital hall & $21 / 12 / 05$ & Self & Time/plain papers \\
\hline $\begin{array}{l}\text { Fact finding on infection } \\
\text { control }\end{array}$ & Maternity & 1.01 .06 & Self & Time \\
\hline Supervisory on records & Maternity & 23.12 .05 & Self & Writing materials \\
\hline Health message & MCH Bay & 23.12 .05 & Self & $\begin{array}{l}\text { Posters and flip } \\
\text { charts }\end{array}$ \\
\hline $\begin{array}{l}\text { Update on the use of } \\
\text { MgS04 }\end{array}$ & Maternity & 23.12 .05 & Self & $\begin{array}{l}\text { Posters and flip } \\
\text { charts }\end{array}$ \\
\hline $\begin{array}{l}\text { Updates on waste } \\
\text { disposal }\end{array}$ & $\begin{array}{l}\text { General } \\
\text { meeting }\end{array}$ & 23.12 .05 & Self & $\begin{array}{l}\text { Stationery and } \\
\text { staff time }\end{array}$ \\
\hline
\end{tabular}




\begin{tabular}{|l|l|l|l|l|}
\hline \multicolumn{5}{|c|}{ Mohamed Kampicha, KRCHN, Moyale District Hospital } \\
\hline ACTIVITY & WHERE & TIME & RESPONSIBILITY & RESOURCE \\
\hline $\begin{array}{l}\text { Feedback on } \\
\text { workshop }\end{array}$ & $\begin{array}{l}\text { Moyale District } \\
\text { Hosp }\end{array}$ & 19.12 .05 & DHEO & Stationery \\
\hline $\begin{array}{l}\text { Supportive } \\
\text { supervision of units } \\
\text { plus dispensaries }\end{array}$ & $\begin{array}{l}\text { MCH and } \\
\text { dispensaries }\end{array}$ & 26.12 .05 & DHEO & Vehicle \\
\hline $\begin{array}{l}\text { Updates on infection } \\
\text { prevention, safe } \\
\text { motherhood }\end{array}$ & $\begin{array}{l}\text { Moyale District } \\
\text { Hospital }\end{array}$ & $\begin{array}{l}3 \text { to } 4^{\text {th }} \\
\text { December } 05\end{array}$ & DHEO & Flip chart, pens \\
\hline $\begin{array}{l}\text { Impact of FGM/C } \\
\text { practices on RH }\end{array}$ & MCH, Maternity & & DHEO & $\begin{array}{l}\text { Hand outs on } \\
\text { topic }\end{array}$ \\
\hline $\begin{array}{l}\text { Routine health } \\
\text { education }\end{array}$ & $\begin{array}{l}\text { Inpatients plus } \\
\text { out patient } \\
\text { department }\end{array}$ & Daily & Teaching aid \\
\hline
\end{tabular}

\begin{tabular}{|l|l|l|l|l|}
\hline \multicolumn{5}{|c|}{ Hambule Mohamed, DPHN, Moyale District Hospital } \\
\hline ACTIVITY & WHERE & TIME & RESPONSIBILITY & RESOURCES \\
\hline Workshop feedback & MDH & 21.12 .05 & DHMT & Time \\
\hline $\begin{array}{l}\text { Identify and distribute } \\
\text { underutilized items in the } \\
\text { store }\end{array}$ & MDH & 22.12 .05 & DPHN & Time \\
\hline $\begin{array}{l}\text { Set up one complete } \\
\text { emergency tray in ANC }\end{array}$ & MDH & 24.12 .05 & DPHN & Time \\
\hline $\begin{array}{l}\text { Conduct on job training } \\
\text { to RH/FP staff on IP and } \\
\text { FGM/C }\end{array}$ & R.H.Fs & $\begin{array}{l}\text { Quarterly } \\
\text { visits }\end{array}$ & DPHN & Time \\
\hline $\begin{array}{l}\text { Calculate UN process } \\
\text { indicators for the district }\end{array}$ & District & Jan 2006 & DPH & Time \\
\hline
\end{tabular}

\begin{tabular}{|l|l|l|l|l|}
\hline \multicolumn{5}{|c|}{ Sora Dima, Moyale District Hospital, District Nutritionist } \\
\hline ACTIVITY & WHERE & TIME & RESPONSIBILITY & RESOURCES \\
\hline Breastfeeding practices & M.C.H & 19.12 .2005 & Nutritionist & None \\
\hline Weaning diet \& time & MCH & 19.12 .05 & Nutritionist & None \\
\hline Attending ward round & M.C.H & 6.1 .06 & Nutritionist & None \\
\hline Feedback to DHMT & & 21.12 .05 & DPHN/DHED/DNO & None \\
\hline $\begin{array}{l}\text { Assessment of weight for age } \\
\text { to children attending MCH }\end{array}$ & MCH & 26.12 .05 & Nutritionist & None \\
\hline
\end{tabular}




\section{GARISSA DISTRICT}

\begin{tabular}{|l|l|l|l|l|}
\hline \multicolumn{4}{|c|}{ A. M. Abdile Xarran, Acting D.C.O, Provincial General Hospital } \\
\hline ACTIVITY & WHERE & TIME & RESPONSIBILITY & RESOURCES \\
\hline Feedback to HMT & PGH & $\begin{array}{l}\text { Dec 05 - } \\
\text { Jan 06 }\end{array}$ & Xarran & None \\
\hline $\begin{array}{l}\text { Strengthening internal } \\
\text { supervision (internal } \\
\text { support) }\end{array}$ & PGH & Jan 06 & Xarran & None \\
\hline $\begin{array}{l}\text { Updating COs on pillars } \\
\text { of SM \& NH }\end{array}$ & PGH & Feb 06 & Xarran & None \\
\hline $\begin{array}{l}\text { CME on management of } \\
\text { FGM/C complication for } \\
\text { health care providers }\end{array}$ & $\begin{array}{l}\text { Garissa VCT } \\
\text { Resource Centre }\end{array}$ & 24.2 .06 & Xarran & None \\
\hline
\end{tabular}

\begin{tabular}{|l|l|l|l|l|}
\hline \multicolumn{5}{|c|}{ Martin Kamau, HRIO, Provincial General Hospital } \\
\hline ACTIVITY & WHERE & TIME & RESPONSIBILITY & RESOURCES \\
\hline $\begin{array}{l}\text { Promote health } \\
\text { education in ANC }\end{array}$ & M.C.H/FP & $\begin{array}{l}\text { Every } \\
\text { morning }\end{array}$ & Kamau & Writing materials \\
\hline $\begin{array}{l}\text { Ensure operational } \\
\text { emergency tray }\end{array}$ & $\begin{array}{l}\text { Labour and } \\
\text { casualty }\end{array}$ & Always & Kamau & $\begin{array}{l}\text { Drugs and } \\
\text { equipment }\end{array}$ \\
\hline Feedback to other staff & PGH & Tuesdays & Kamau & None \\
\hline $\begin{array}{l}\text { Ensure availability of } \\
\text { stationeries eg } \\
\text { partograph }\end{array}$ & PGH & Tuesdays & Kamau & Duplicating paper \\
\hline
\end{tabular}

\begin{tabular}{|l|l|l|l|l|}
\hline \multicolumn{5}{|c|}{ Warfa Osman, DMOH, MOHs Office } \\
\hline ACTIVITY & WHERE & TIME & RESPONSIBILITY & RESOURCES \\
\hline $\begin{array}{l}\text { Feedback from } \\
\text { workshop }\end{array}$ & $\begin{array}{l}\text { District } \\
\text { headquarters }\end{array}$ & 13.12 .05 & DHMT & None \\
\hline Roll out FANC & PGH & Jan 2006 & DMOH, DPHN & Ksh. 224,200 \\
\hline Roll out EMOC QA & Garissa hospitals & $\begin{array}{l}\text { Jan to Feb } \\
2006\end{array}$ & Warfa, All DMOHs & Ksh. 152,000 \\
\hline $\begin{array}{l}\text { Sensitize and } \\
\text { distribute national RH } \\
\text { guidelines to all } \\
\text { facility incharges }\end{array}$ & $\begin{array}{l}\text { Health centers and } \\
\text { dispensaries }\end{array}$ & Dec 05 & DMOH, DPHN & Ksh. 100,00 \\
\hline $\begin{array}{l}\text { Pilot output based } \\
\text { approach (OBA) in 3 } \\
\text { facilities }\end{array}$ & $\begin{array}{l}\text { Balambala, Daadab, } \\
\text { Modogashe }\end{array}$ & $\begin{array}{l}\text { Nov. 05 - } \\
\text { March 06 }\end{array}$ & $\begin{array}{l}\text { DMOH, DPHN, } \\
\text { UNICEF, Facility } \\
\text { incharges }\end{array}$ & Ksh.400,000 \\
\hline
\end{tabular}




\begin{tabular}{|l|l|l|l|l|}
\hline \multicolumn{5}{|c|}{ Mohamed Hussein Ibrahim, Nurse, PGH Garissa } \\
\hline ACTIVITY & WHERE & TIME & RESPONSIBILITY & RESOURCES \\
\hline $\begin{array}{l}\text { Feedback from workshop } \\
\text { RH, SMH through CME } \\
\text { session }\end{array}$ & PGH staff & 13.12 .05 & Hussein & None \\
\hline $\begin{array}{l}\text { Meeting with staff on RH } \\
\text { update }\end{array}$ & $\mathrm{MCH}$ & $20 / 12 / 05$ & Hussein & None \\
\hline $\begin{array}{l}\text { Implement best practice i.e } \\
\text { FANC, IP, Post Natal, } \\
\text { PMCTC }\end{array}$ & $\mathrm{MCH}$ & $2 / 1 / 06$ & Hussein & None \\
\hline $\begin{array}{l}\text { Give health talk on } \\
\text { RH/FGM/C }\end{array}$ & $\mathrm{MCH}$ & $14 / 1 / 06$ & Hussein & None \\
\hline Update emergency tray & MCH & $19 / 12 / 05$ & Hussein & None \\
\hline $\begin{array}{l}\text { Strengthen MDR in the } \\
\text { hospital }\end{array}$ & Maternity & $3 / 01 / 06$ & Hussein & None \\
\hline
\end{tabular}

\begin{tabular}{|l|l|l|l|l|}
\hline \multicolumn{5}{|c|}{ Mohammed Salat Dagane, PNO, PMOs Office Garissa } \\
\hline ACTIVITY & WHERE & TIME & RESPONSIBILITY & RESOURCES \\
\hline Feedback to PHMT & PMOs Office & 14.12 .05 & PNO & None \\
\hline $\begin{array}{l}\text { Supervisory visits to } \\
\text { districts }\end{array}$ & $\begin{array}{l}\text { Garissa } \\
\text { District }\end{array}$ & 7.1 .06 & PHMT & None \\
\hline $\begin{array}{l}\text { Supervisory visits to } \\
\text { districts }\end{array}$ & Ijara District & $20 / 1 / 06$ & PHMT & $\begin{array}{l}\text { Ksh. 150,000 for fuel } \\
\text { and allowances }\end{array}$ \\
\hline $\begin{array}{l}\text { Supervisory visits to } \\
\text { districts }\end{array}$ & Wajir District & Dec 05 & DMOH, DPHN & $\begin{array}{l}\text { Ksh. 210,000 for fuel } \\
\text { and allowances }\end{array}$ \\
\hline $\begin{array}{l}\text { Supervisory visits to } \\
\text { districts }\end{array}$ & $\begin{array}{l}\text { Mandera } \\
\text { District }\end{array}$ & $\begin{array}{l}\text { Nov. 05 - } \\
\text { March 06 }\end{array}$ & $\begin{array}{l}\text { DMOH, DPHN, } \\
\text { UNICEF, Facility } \\
\text { incharges }\end{array}$ & $\begin{array}{l}\text { Ksh.345,000 for fuel } \\
\text { and allowances }\end{array}$ \\
\hline
\end{tabular}

\begin{tabular}{|l|l|l|l|l|}
\hline \multicolumn{5}{|c|}{ Dakane Khalif, DPHN, DPHN Office Garissa } \\
\hline ACTIVITY & WHERE & TIME & RESPONSIBILITY & RESOURCES \\
\hline $\begin{array}{l}\text { Give feedback to other DHMT } \\
\text { members }\end{array}$ & DMOH office & 16.12 .05 & Dakane & None \\
\hline $\begin{array}{l}\text { Stores search of equipment of } \\
\text { delivery }\end{array}$ & Stores & 19.12 .05 & Dakane & None \\
\hline $\begin{array}{l}\text { Sensitization of health workers } \\
\text { in rural facilities about the } \\
\text { training }\end{array}$ & Rural Facilities & 29.12 .05 & Dakane & None \\
\hline $\begin{array}{l}\text { Do H/count for instruments in } \\
\text { the facilities }\end{array}$ & Rural facilities & 24.12 .05 & Dakane & None \\
\hline $\begin{array}{l}\text { Share with neighbouring } \\
\text { facilities on FGM/C dangers and } \\
\text { Islamic views }\end{array}$ & Rural facilities & 13.12 .05 & Dakane & None \\
\hline
\end{tabular}




\begin{tabular}{|c|c|c|c|c|}
\hline \multicolumn{5}{|c|}{ Hussein Aden, DMLT, DMLT Office Garissa } \\
\hline ACTIVITY & WHERE & TIME & RESPONSIBILITY & RESOURCES \\
\hline $\begin{array}{l}\text { Give feedback on } \\
\text { reproductive health } \\
\text { to other DHMT and } \\
\text { lab staff }\end{array}$ & DMOH office & 13.12 .05 & DMLT & None \\
\hline $\begin{array}{l}\text { Strengthen infection } \\
\text { prevention by } \\
\text { introducing CME at } \\
\text { PGH }\end{array}$ & $\begin{array}{l}\text { PGH Garissa, } \\
\text { IFTIN sub } \\
\text { district hospital }\end{array}$ & 15.12 .05 & DMLT & None \\
\hline $\begin{array}{l}\text { To avoid expiry of } \\
\text { blood by use of FIFO } \\
\text { system }\end{array}$ & PGH & $\begin{array}{l}\text { Every Thursday } \\
\text { morning }\end{array}$ & $\begin{array}{l}\text { DMCT and PGH lab in } \\
\text { charge }\end{array}$ & None \\
\hline $\begin{array}{l}\text { Give health talks on } \\
\text { reproductive and } \\
\text { FGM/C to my } \\
\text { neighborhood }\end{array}$ & Home & $\begin{array}{l}\text { Twice in a } \\
\text { month i.e } \\
\text { Sunday night }\end{array}$ & $\begin{array}{l}\text { DMLT and any } \\
\text { volunteer }\end{array}$ & None \\
\hline
\end{tabular}




\section{Appendix 2. Time Table}

\begin{tabular}{|c|c|c|c|}
\hline Day & Time & Activity & Facilitator \\
\hline \multirow{15}{*}{ DAY 1} & 08.00-08.30am & Registration & Dr. Ahmed/Secretariat \\
\hline & 8.30-8.40am & Introductions & DANIDA/Dr. Ahmed \\
\hline & $8.40-8.50 \mathrm{AM}$ & Expectations/Norms & UNICEF/Siyat \\
\hline & $8.50-9.40 \mathrm{am}$ & Opening remarks by $\mathrm{PMO}$ & $\begin{array}{l}\text { PMO, DRH, DANIDA, Pop } \\
\text { Council }\end{array}$ \\
\hline & 9.40-10.00am & Pretest & Jaldesa/ Hambule/Siyat \\
\hline & 10.00-10.30am & Overview of FGM/C activities in NEP & UNICEF \\
\hline & 10.30-11.00am & TEA/COFFEE BREAK & \\
\hline & $11.00-11.15$ & Workshop objectives & DRH/Jaldesa/Hambule \\
\hline & $11.15-12.00$ & RH Policy \& Kenya SM model & Prof. Karanja / Salat \\
\hline & $12.00-1.00 \mathrm{pm}$ & Infection prevention & Adam / Prof. Karanja \\
\hline & $1.00-2.00 \mathrm{pm}$ & LUNCH BREAK & \\
\hline & 2.00-2.30PM & Emergency preparedness \& referral & Dr. Jaldesa/ Siyat \\
\hline & $2.30-4.00 \mathrm{PM}$ & IPCC Lecture and Video & Adam/Prof. Karanja \\
\hline & $4.00-4.30 \mathrm{pm}$ & TEA/COFFEE BREAK & \\
\hline & $4.30-6.00 \mathrm{pm}$ & $\begin{array}{l}\text { Video shows (Safe Motherhood, Mama Song, } \\
\text { Infection Prevention) }\end{array}$ & Dr. Jaldesa/Dr. Ahmed \\
\hline \multirow{9}{*}{ DAY 2} & 8.00-9.00am & Data collection \& Utilization & Adam \\
\hline & 9.00-9.45am & $\begin{array}{l}\text { MDR Lecture/ video show on beyond the } \\
\text { numbers, my sister my self }\end{array}$ & Siyat \\
\hline & 9.45-10.30am & Introduction to FGM/C & Jaldesa/Salat \\
\hline & 10.30-11.00am & TEA/COFFEE BREAK & \\
\hline & $11.00-12.00$ & $\begin{array}{l}\text { Immediate and long term complications } \\
\text { associated with FGM/C }\end{array}$ & Jaldesa/Ahmed \\
\hline & $12.00-1.00 \mathrm{pm}$ & $\begin{array}{l}\text { Management of FGM/C complications } \\
\text { Video show road to change (FGM/C) }\end{array}$ & Jaldesa/Ahmed \\
\hline & $1.00-2.00 \mathrm{pm}$ & LUNCH BREAK & \\
\hline & $2.00-5.00 \mathrm{pm}$ & FANC & Prof. Karanja/Siyat \\
\hline & $5.30-6.00 \mathrm{pm}$ & ANC in Type I, II, III, IV FGM/C & Dr. Jaldesa/Hambule \\
\hline \multirow{11}{*}{ DAY 3} & 8.00-9.30am & Management of normal labour & Prof. Karanja /Siyat + (TEAM) \\
\hline & 9.30-10.30am & $\begin{array}{l}\text { Management of labour in presence of FGM/C } \\
\text { I, II, III, IV }\end{array}$ & Jaldesa/Hambule \\
\hline & 10.30-11.00am & TEA/C OFFEE BREAK & \\
\hline & $11.00-1.00 \mathrm{pm}$ & Introduction to Clinical Areas & TEAM \\
\hline & & Groups Reports From Clinical Areas & TEAM \\
\hline & $1.00-2.00 \mathrm{pm}$ & LUNCH BREAK & \\
\hline & $2.00-3.30 \mathrm{pm}$ & Partograph in Labour & Jaldesa/Ahmed \\
\hline & $3.30-4.00 \mathrm{pm}$ & Pre-eclampsia/Eclampsia & Dr. Ahmed \\
\hline & $4.00-430$ & Malaria/Anemia in pregnancy & Salat \\
\hline & $4.30-5.00$ & TEA BREAK & \\
\hline & $5.00-600$ & HIV in pregnancy & Adam/Ahmed \\
\hline
\end{tabular}




\begin{tabular}{|c|c|c|c|}
\hline \multirow{9}{*}{ DAY 4} & 8.30-12.00noon & CLINICAL EXPERIENCE & TEAM \\
\hline & & Reports from Clinical areas & TEAM \\
\hline & & LUNCH BREAK & \\
\hline & \multirow{3}{*}{$2.00-4.30 \mathrm{pm}$} & Care of normal Neonate & KHADIJA/Hambule \\
\hline & & Neonatal Asphyxia/resuscitation & Dr. KHADIJA/Siyat \\
\hline & & Neonatal Sepsis & Dr. KHADIJA/Salat \\
\hline & $4.30-5.00 \mathrm{pm}$ & TEA/COFFEE BREAK & \\
\hline & $5.00-6.00 \mathrm{pm}$ & Focused Postpartum care & Dr Ahmed/Prof. Karanja \\
\hline & & PPH, puerperal sepsis & Dr Ahmed \\
\hline \multirow{8}{*}{ DAY 5} & 8.30 - 11.00am & Clinical Experience & TEAM \\
\hline & 11am -1pm & PRAYERS & \\
\hline & $1.00 \mathrm{pm}-2 \mathrm{pm}$ & LUNCH BREAK & \\
\hline & $2.00-3.00 \mathrm{pm}$ & Prolonged Labor \& Obstructed labor & Ahmed/ Prof. Karanja \\
\hline & $3.00-4.00 \mathrm{pm}$ & Role of HC Providers in abandonment of FGM/C & Jaldesa/DRH/Adam \\
\hline & $4.00-4.30 \mathrm{pm}$ & Human Rights and Legal implications of FGM/C & Jaldesa/DRH/Adam \\
\hline & $4.30-5.00 \mathrm{pm}$ & TEA Break & \\
\hline & $5.00-6.00 \mathrm{pm}$ & RVF/VVF; role of FGM/C & Dr. Ahmed \\
\hline \multirow[b]{8}{*}{ DAY 6} & $7.30-12.00$ & Clinical Experience & TEAM \\
\hline & $12-1.00 \mathrm{pm}$ & Reports from Clinical areas & TEAM \\
\hline & $1.00-2.00 \mathrm{pm}$ & LUNCH BREAK & \\
\hline & $2.00-4.30 \mathrm{pm}$ & Religious perspective of FGM/C & Sheikh \\
\hline & $4.30-5.00 \mathrm{pm}$ & Introducing National plan of action on FGM/C & Adam \\
\hline & $5.00-5.45 \mathrm{pm}$ & TEA/COFFEE BREAK & \\
\hline & $5.45-6.00 \mathrm{pm}$ & FP UPDATES & Hambule \\
\hline & & $\begin{array}{l}\text { Action Plan and activities by HC providers in their } \\
\text { facilities }\end{array}$ & $\begin{array}{l}\text { JALDESA/ } \\
\text { UNICEF/DANIDA } \\
\text { Team }\end{array}$ \\
\hline \multirow{7}{*}{ DAY 7} & $8.30-9.30 \mathrm{am}$ & Post test & Salat/Jaldesa \\
\hline & 9.00-10.30am & Facilities Records Reports & Jaldesa/Adam (TEAM) \\
\hline & $10.30-11.00$ & TEA/COFFEE BREAK & \\
\hline & $11.00-1.00 \mathrm{pm}$ & $\begin{array}{l}\text { Facility/ Clinic strengthening/ individual plans } \\
\text { (what is needed, what can be done, who will, how) }\end{array}$ & $\begin{array}{l}\text { Hambule/Jaldesa } \\
\text { (TEAM) }\end{array}$ \\
\hline & $1.00-2.00 \mathrm{pm}$ & LUNCH BREAK & \\
\hline & $2.00-2.30 \mathrm{pm}$ & Review the individual action plans. & Jaldesa (TEAM) \\
\hline & $2.30-5.00 \mathrm{pm}$ & CLOSING & $\begin{array}{l}\text { PMO/DANIDA/ } \\
\text { UNICEF }\end{array}$ \\
\hline
\end{tabular}




\section{Day One, Session 1: Introduction and overview of reproductive health policy, including safe motherhood and newborn health}

\section{LEARNING OBJECTIVES}

At the end of the session, health workers will:

1. Describe the overview of reproductive health components and policy.

2. Define Safe Motherhood and Newborn Health

3. Describe Safe Motherhood and Newborn Health

4. Identify the key elements of Safe Motherhood and Newborn Health

5. Identify the factors and causes of maternal and perinatal morbidity and mortality

6. Explain the Millennium Development Goals relevant to SMNH

7. Explain basic and comprehensive Essential Obstetric Care

\section{TRAINING NEEDS}

\section{Knowledge required:}

i. Understanding reproductive health components and policy.

ii. Understanding Safe Motherhood and Newborn Health

iii. Appreciates magnitude of the problem

iv. Key elements of SMNH

v. Immediate and underlying factors and causes of maternal deaths in Kenya

vi. Understand the Millennium Development Goals

vii. Strategies for implementing SMNH in your area of work

viii. Challenges for implementing Safe Motherhood and Newborn Health

\section{Skills required:}

i. Implementations of reproductive health policy in the districts

ii. Compilation of maternal and perinatal morbidity and mortality data

iii. Analysis of maternal and perinatal deaths near misses: maternal and perinatal audit

iv. Analysis of factors and causes contributing to maternal deaths

v. Formulation of strategies for reducing avoidable factors of maternal deaths in the area. 
vi. Outlining strategies for implementing SMIH

vii. Advocacy: Information, Education, Communication (IEC) on Safe Motherhood issues

viii. Monitoring and Evaluation of progress

\section{Attitude required:}

Health care providers should appreciate that most maternal and perinatal deaths are avoidable. In spite of maternal and child health/family planning programmes, maternal and newborn health must be given its due prominence. Safe Motherhood is a basic human right as women are entitled to enjoy a safe pregnancy and childbirth.

\section{Session 2: Data collection and utilisation and its role in monitoring and evaluation}

\section{LEARNING OBJECTIVES}

By the end of the session, health care providers will:

1. Define monitoring, review and evaluation

2. Describe types of records used to collect maternal and perinatal service data

3. Describe the UN obstetric indicators and

4. Explain the importance of maternal and perinatal data collection and record keeping

5. Describe 'on site' review and evaluation of data

6. Utilize maternal and perinatal service data for planning.

7. Appreciate the importance of regular facility meetings to discuss reproductive health issues

\section{TRAINING NEEDS}

Knowledge required:

i. Understand monitoring, review and evaluation

ii. Types of records and their use

iii. Understand the use of UN and national indicators

iv. Understand how to calculate basic rates, ratios and proportions for selected indicators

v. Importance of data collection, storage 
vi. Mechanisms for retrieval and analysis of data

vii. Report writing and feedback

\section{Skills required:}

i. Accurate documentation of different records and registers

ii. Coaching and supervision of data collection and management

iii. Safe storage and easy retrieval of records

iv. Regular and accurate compilation of records

v. Analysis and report writing

vi. Dissemination of maternal and perinatal health service data

\section{Attitude required:}

Health care providers will appreciate data collection, management analysis report writing appreciate the importance of monitoring review and evaluation, which includes supervision of record keeping, recording information correctly, and the relevance of various records used in providing appropriate high quality care. Health facility management committees should hold regular meetings to review the reproductive health data and focus on the issues rather than fault-finding.

\section{Session 3: Interpersonal communication and counselling LEARNING OBJECTIVES}

At the end of the session, health care providers will:

1. Define terms used in interpersonal communication and counselling (IPCC)

2. Identify health activities that require IPCC

3. Explain the basic counselling principles and IPCC skills and techniques

4. Explain the purpose of counselling

5. Describe the qualities of a good counsellor

6. Explain the counselling process utilizing GATHER steps

7. Identify factors that facilitate and those that interfere with counselling

8. Review elements of quality care 


\section{TRAINING NEEDS}

\section{Knowledge required:}

i. Terms used in IPCC

ii. The basic counselling principles

iii. IPCC skills and techniques

iv. Counselling process using GATHER

v. Qualities of a good counsellor

vi. Factors that facilitate or interfere with counselling

vii. Elements of quality of care

\section{Skills required:}

i. Demonstrate skills, qualities and techniques of a good counsellor

ii. Demonstrate utilization of GATHER in the counselling process

iii. Ability to identify positive and negative factors that influence counselling

\section{Attitude required:}

Practice of IPCC skills is necessary to foster a positive relationship with individual clients and community members especially when interacting with clients attending maternal health services. Maternal health care should be personalised and requires excellent interpersonal skills since listening to clients is just as important as giving advice.

\section{Session 4: Infection Prevention}

\section{LEARNING OBJECTIVES}

At the end of the session, health care providers will:

1. Define Infection Prevention

2. Describe the disease transmission cycle

3. Explain the purpose of infection prevention

4. Explain the principles of infection prevention

5. Describe the basic infection prevention methods

6. Describe the procedures used to process instruments

7. Describe the procedures for waste disposal 


\section{TRAINING NEEDS}

\section{Knowledge required:}

i. Definition of infection prevention

ii. Purpose of infection prevention

iii. Knowledge on disease transmission cycle

iv. Explanation of the IP principles

v. Basic infection prevention methods

vi. Procedures used to process equipment vii. How to make a chlorine solution

\section{Skills required:}

i. Implementation of Infection Prevention measures

ii. Demonstrate appropriate infection prevention practices at all times.

\section{Attitude required:}

Infection prevention practice will go along way to reduce transmission within the health facility. This has an impact on the quality of care a woman will receive.

\section{Day Two Session One: Emergency Preparedness}

\section{LEARNING OBJECTIVES}

At the end of the session, health care provider will:

1. Define Emergency Preparedness

2. List the qualities required for handling an emergency

3. Describe the elements of emergency preparedness

4. Prepare a complete emergency trolley/tray and equipment

5. Describe the management of an emergency

6. Describe the preparation for a referral

7. Explain the documentation process during and after an emergency 


\section{TRAINING NEEDS}

\section{Knowledge required:}

i. Definition of emergency preparedness

ii. Understand the process of emergency preparedness

iii. Items required for emergency preparedness

iv. Criteria and response for handling obstetric emergencies

\section{Skills required:}

i. Implementation of emergency preparedness measures

ii. Adult and newborn resuscitation

iii. Midwifery skills

\section{Attitude required:}

Health care providers should appreciate the importance of emergency preparedness as an essential part of the daily routine. There should be willingness and teamwork to ensure that everyone in the facility knows their roles to respond efficiently to emergencies.

\section{Rationale:}

Emergency preparedness and appropriate response saves lives. Most emergencies in SMNH are generally unpredictable thus the need for preparedness at all times.

\section{Session Two: Introduction to FGM/C}

\section{LEARNING OBJECTIVES}

1. To define and classify FGM/C

2. To describe the prevalence of FGM/C in Kenya and Africa

3. To describe why different communities practice FGM/C

4. To describe the Short and long term physical complications of FGM/C

5. To describe the Psychosocial and sexual complications of FGM/C

6. Understand the management of different types of FGM/C complications 
The expected outcomes for this are as follows;

\section{Knowledge:}

By the end of the training, the participants will be able to:

1. To describe the different types of FGM/C and the complications of the practice.

\section{Skills:}

By the end of the one weeks training, the participants will be able to:

1. Understand why different communities practice FGM/C

2. Understand the health complications associated with the FGM/C

\section{Day Three Session 1: Focused Antenatal Care LEARNING OBJECTIVES}

At the end of the session, health care providers will:

1. Define antenatal care.

2. Describe focused antenatal care

3. Explain the reason for focused antenatal care

4. Take history from and perform physical examination of a pregnant woman

5. Assist the pregnant woman to develop and implement a birth plan

6. Explain the possible complications during pregnancy, labour, delivery and postpartum period

7. Manage or refer women with high risk conditions or complications

8. Screen and manage appropriately for reproductive tract infections (RTI) in pregnancy.

9. Demonstrate Health Promotion activities including anti FGM/C messages during antenatal period.

10. To describe problems associated with FGM/C during pregnancy.

11. To explain the management of women with Type I, II, IV FGM/C during pregnancy.

12. To state the indications for and timing of opening up Type III FGM/C

13. To discuss the procedure of opening up Type III FGM/C during pregnancy.

14. To describe the post care of an opened Type III FGM/C during pregnancy. 


\section{TRAINING NEEDS}

\section{Knowledge required}

i. Understand antenatal care.

ii. Understand focused antenatal care

iii. Reason for focused antenatal care

iv. History taking and physical examination of a pregnant woman

v. Development of an individualized birth plan

vi. Understand the use of Syndromic Chart for RTI management.

vii. Complications of pregnancy associated with FGM/C

\section{Skills required}

i. History taking and physical examination of a pregnant woman

ii. Ability to implement appropriate management or timely referral

iii. Demonstrate IPCC in providing antenatal care

\section{Attitude required}

Health care providers to appreciate the importance of focused antenatal care in SMNH.

\section{Session 2: Malaria in Pregnancy}

\section{LEARNING OBJECTIVES}

At the end of the session, health care providers will:

1. Define malaria

2. Explain the clinical features of malaria

3. Explain the effects of malaria in pregnancy

4. Explain intermittent preventive treatment of malaria in pregnancy

5. Describe treatment of severe or complicated malaria in adults

6. Explain relationship of malaria and HIV in pregnancy

7. Describe other ways of preventing malaria 


\section{TRAINING NEEDS}

Knowledge required:

i. Definition, causes, classification, types and diagnosis of malaria

ii. Identification of risk factors in malaria

iii. Guidelines for Intermittent Preventive Treatment for malaria

iv. Guidelines for treatment of Insecticide Treated Nets

v. Management of severe and complicated malaria in adults

\section{Skills required:}

i. Diagnosis of malaria

ii. Assessment of malaria during antenatal period

iii. Management of uncomplicated malaria in pregnancy

iv. Criteria for referring woman with severe malaria

\section{Attitude required}

- North Eastern Province is one of the regions in Kenya with malaria throughout the year. Health care providers must be aware of the seriousness of malaria and should ensure early treatment of malaria in pregnant women to prevent complications.

- Develop a positive relationship with the pregnant woman in devising ways to prevent malaria including the importance of Intermittent Preventive Treatment of malaria and Insecticide Treated Nets.

\section{Session 3: Anaemia in Pregnancy}

\section{LEARNING OBJECTIVES}

At the end of the session, health care providers will:

1. Define and classify anaemia

2. Describe common causes of anaemia

3. Identify women at risk of developing anaemia

4. Diagnose anaemia

5. Explain effects of anaemia in pregnancy

6. Explain the management of anaemia during pregnancy and labour 


\section{TRAINING NEEDS}

\section{Knowledge required:}

i. Definition, causes, classification, types and diagnosis of anaemia

ii. Identification of risk factors in anaemia

iii. History taking and patient examination procedures

iv. Management of anaemia in pregnancy, labour and the puerperium

v. When, where and how to refer

\section{Skills required:}

i. Diagnosis of anaemia in pregnancy

ii. Ascertaining the causes of anaemia in pregnancy

iii. Management of a woman with anaemia antenatally, intrapartum and postpartum

iv. Criteria for referral

\section{Attitude required:}

There is high prevalence of anaemia in the region. The commonest cause of the anaemia is malnutrition and one secondary to malaria. Health care providers use good interpersonal skills to communicate successfully the line of treatment for a case of anaemia to the patient and her relatives. The importance of antenatal care in identification and treatment of anaemia must be emphasized.

\section{Session 4: Pre- Eclampsia and Eclampsia}

\section{LEARNING OBJECTIVES}

At the end of the session, health care provider will:

1. Define pre-eclampsia and eclampsia

2. Identify risk factors for pre-eclampsia and eclampsia

3. Diagnose and classify pre-eclampsia

4. Diagnose eclampsia

5. Manage a woman with pre-eclampsia and eclampsia

6. Referral 


\section{TRAINING NEEDS}

Knowledge required:

1. Definition of pre-eclampsia and eclampsia

2. Understand the epidemiology of pre-eclampsia and eclampsia

3. Clinical features of pre-eclampsia/eclampsia

4. Diagnosis of pre-eclampsia and eclampsia

5. Management of pre-eclampsia/eclampsia

6. The referral procedure

\section{Skills required:}

i. Patient examination and evaluation procedures

ii. Correct technique of blood pressure measurement

iii. Correct technique of urine dipstick analysis and interpretation

iv. Correct administration and monitoring of Magnesium Sulphate (MgSO4) or diazepam where $\mathrm{MgSO} 4$ is not available

v. Correct administration and monitoring of antihypertensive (Hydrallazine and/or Nifedipine)

vi. Correct referral procedures

\section{Attitude required:}

Health care providers should appreciate the importance of pre-eclampsia and eclampsia and urgency and diligence in dealing with these conditions. They should ensure that the patient and her relatives understand that pre-eclampsia/ eclampsia are serious and life threatening conditions.

\section{Session 5: HIV in Pregnancy}

\section{Learning Objectives:}

At the end of the session, health care providers will:

1. Describe the magnitude of HIV in pregnancy in Kenya

2. Describe the magnitude of mother to child transmission of HIV in Kenya

3. Describe the management of HIV positive women during pregnancy, labour, and postpartum period

4. Describe baby care and feeding alternatives for babies of HIV infected mothers

5. Outline anti-retroviral therapy in HIV infection 


\section{TRAINING NEEDS}

\section{Knowledge required:}

i. Magnitude of HIV in pregnancy and mother to child transmission of HIV

ii. Antenatal, intrapartum and postpartum care in HIV infected women

iii. Outline of infant feeding options in HIV infected women

iv. Anti-retroviral drug therapy regimes

\section{Skills required:}

i. Identification of HIV infection in pregnant women

ii. Provision of care to HIV infected women antenatal, intrapartum and postpartum

iii. Choice of infant feeding options and antiretroviral drug therapy

\section{Attitude required:}

Though the prevalence of HIV in this region is low, the health care providers must realize the importance of HIV infection and the need for action. Good counseling skills are important to combat the psychological aspects of HIV infection in pregnancy. Special attention is given to nutrition, risk reduction behaviour, counselling on infant feeding and family planning services. Changes in midwifery and obstetric practice are required due to HIV infection in pregnancy.

\section{DAY 4: Management of normal labour and delivery}

\section{Learning Objectives:}

At the end of the session, the health care providers will:

1. Define and make a diagnosis of normal labour

2. Assess the condition of the patient and foetus as well as the stage of labour (assess progress of labour)

3. Manage normal labour at different stages

4. Define and describe the use of a partogram

5. Identify signs of abnormal labour

6. Take appropriate action for abnormal labour including referral

7. Establish and maintain rapport with woman in labour

8. To describe problems associated with FGM/C during labour, delivery, and postpartum 
9. To explain the management of women with Type I, II, IV FGM/C during labour, delivery and postpartum.

10. To state the indications and timing for opening up Type III FGM/C

\section{TRAINING NEEDS}

\section{Knowledge required:}

i. Signs and symptoms of the different stages of normal labour

ii. General, abdominal and pelvic examination in normal labour

iii. Management of normal labour at different stages

iv. The use of the partogram and its components

v. Identify signs of abnormal labour and

vi. Understand when to take appropriate action including referral

vii. Understand psycho-social support women need during labour

\section{Skills required:}

i. Diagnosis of normal labour

ii. Assessment of patient's condition and progress of labour

iii. Management of normal labour at different stages

iv. Fill in the partograph using appropriate symbols

v. Correct interpretation of the partograph

vi. Based on available resources and information take appropriate action.

vii. Demonstrate good interpersonal communication especially with infibulated women.

\section{Attitude required:}

Health care providers should appreciate the importance of monitoring labour using appropriate tools and early recognition of abnormal signs. In case of referral, explain the reason, urgency and place for referral.

Every woman in labour should be treated with dignity and respect and a right to privacy and confidentiality at all times. All women should be given the choice of having a companion (partner, friend or relative) during labour and childbirth. 


\section{Session 2: Postpartum Care}

\section{Learning Objectives:}

At the end of the session, the health care provider will:

1. Define the puerperium/ post partum / post natal period

2. State the aims and timing of post partum care

3. Explain the signs and symptoms of normal post partum period

4. Manage normal puerperium

5. Identify complications/danger signs of postpartum period

6. Counsel the mother on personal hygiene, safer sex, nutrition, breastfeeding and family planning.

\section{TRAINING NEEDS}

\section{Knowledge required:}

i. Definition of puerperium/post partum/post natal period

ii. Aims and timing of the post natal care

iii. Signs and symptoms of normal puerperium

iv. Management of normal puerperium

v. Complications/danger signs of postpartum period

vi. Counselling the mother on personal hygiene, safer sex, nutrition, breastfeeding and family planning.

\section{Skills required:}

i. Ability to recognise and manage normal puerperium

ii. Ability to recognise and manage complications of puerperium

iii. Ability to counsel a post partum mother

\section{Attitude required:}

The health care provider should appreciate the importance of post partum care in reducing maternal and perinatal morbidity and mortality. 


\section{Session 3: Management of postpartum haemorrhage}

\section{Learning objectives:}

At the end of the session, the health care provider will:

1. Define Primary and Secondary Postpartum Haemorrhage

2. Identify predisposing factors of Primary Postpartum Haemorrhage

3. FGM/C as a risk factor for $\mathrm{PPH}$

4. Assess a patient with Primary Postpartum Haemorrhage

5. Manage a woman with Primary Post Partum Haemorrhage

6. Manage woman who requires manual removal of placenta

7. Organize appropriate referral of a patient with Primary Post Partum Haemorrhage from a rural health facility

8. Identify predisposing factors of Secondary Postpartum Haemorrhage

9. Assess a patient with Secondary Postpartum Haemorrhage

10. Manage a woman with Secondary Post Partum Haemorrhage

\section{TRAINING NEEDS}

Knowledge required:

i. Definition of Postpartum Haemorrhage

ii. Diagnosis, causes and pre disposing factors of $\mathrm{PPH}$

iii. Assessment of condition of a patient with Postpartum Haemorrhage

iv. Management of a woman with PPH

v. Management of a woman who requires manual removal of placenta

vi. Appropriate referral of a patient with $\mathrm{PPH}$

\section{Skills required:}

i. $\quad$ Ability to diagnose Post Partum Haemorrhage (PPH)

ii. Ability to assess the condition of a patient with $\mathrm{PPH}$

iii. Examination of placenta and membranes

iv. Active management of $\mathrm{PPH}$

v. Competency in manual removal of placenta

vi. Appropriate referral procedures 


\section{Attitude required:}

Health care providers should appreciate that any excessive bleeding during the puerperium is critical. It is especially important that the patient is closely observed during the first few hours following delivery. Health care providers will communicate successfully the diagnosis of PPH and be able to generate the cooperation of the patient and her relatives in arranging blood for the patient.

\section{Session 4: Puerperal sepsis}

\section{Learning objectives:}

At the end of the session, the health care provider will:

1. Define puerperal sepsis and puerperal infection

2. Identify predisposing of factors for puerperal sepsis

3. Diagnose puerperal sepsis

4. Manage puerperal sepsis

5. Identify complications of puerperal sepsis

6. Arrange for appropriate referral

\section{TRAINING NEEDS}

\section{Knowledge required:}

i. Definition of puerperal sepsis

ii. Identification of risk factors of puerperal sepsis

iii. Diagnosis of puerperal sepsis

iv. Management of woman with puerperal sepsis

v. State complications of puerperal sepsis

vi. When and where to refer patients with history and treatment notes

\section{Skills required:}

i. Understanding of puerperal sepsis

ii. Management of puerperal sepsis

iii. Appropriate procedures for referral 


\section{Attitude required:}

Health care provider should appreciate the importance of prompt action when a woman presents with fever or sepsis or both. The predisposing factors for puerperal sepsis must be discussed with the patient and their relatives and ensure they understand the necessary treatment.

\section{Session 5: Obstructed labour - rupture of the uterus}

\section{Learning objectives:}

At the end of the session, health care provider will:

1. Define obstructed labour

2. Explain causes of obstructed labour

3. Diagnose obstructed labour

4. Describe complications of obstructed labour

5. Manage a woman with obstructed labour

6. Define ruptured uterus

7. Describe the predisposing factors for ruptured uterus

8. Diagnose ruptured uterus

9. Manage a woman with ruptured uterus

10. Describe referral procedure

\section{TRAINING NEEDS}

\section{Knowledge required:}

i. Clinical features of obstructed labour

ii. Clinical features of ruptured uterus

iii. History taking and patient examination procedures

iv. Patient evaluation procedures

v. Indications of vacuum extractor, laparotomy, caesarean section, etc

vi. Indications of oxytocics, antibiotics, pain relief in labour

vii. When, where and how to refer a patient with referral notes 


\section{Skills required:}

i. History taking and patient evaluation procedures

ii. Diagnosis of obstructed labour/ruptured uterus

iii. Ascertain the causes of obstructed labour/ruptured uterus

iv. Assess patient's condition to see if vaginal delivery is possible

v. Conducting assisted vaginal delivery by using forceps/vacuum extractor

vi. Criteria for referral

vii. IPCC skills to inform patient and relatives reasons for management and /or referral

\section{Attitude required:}

Health care providers will use counselling skills to communicate the management and treatment for a woman with obstructed labour or ruptured uterus to the patient and her relatives. Emergency preparedness is extremely important to reduce the risks of maternal and perinatal morbidity and mortality.

\section{Day 5. Session 1: Neonatal care/care of a healthy newborn}

\section{Learning Objectives:}

At the end of the session, health care provider will:

1. Define a newborn infant

2. Explain the immediate care of the newborn baby

3. Assess the condition of the normal newborn

4. Explain the subsequent care of the newborn baby

5. Manage the normal newborn

\section{TRAINING NEEDS}

\section{Knowledge required:}

i. Clinical features of the normal newborn

ii. The parameters of the APGAR score

iii. Recognise the danger signs

iv. When and where to send a newborn infant with referral notes 


\section{Skills required:}

i. Physical examination of the baby

ii. Determine the clinical features of the normal newborn

iii. Management of the normal newborn

iv. Counselling of mothers on new born and infant care

\section{Attitude required:}

Interpersonal communication and counselling skills are important to enable new mothers to feel confident in caring for their newborn babies. Information on potential problems and key areas to observe must be highlighted.

\section{COMPLICATIONS OF NEONATAL PERIOD}

\section{Session 2: Asphyxia neonatorum and new born resuscitation}

\section{Learning objectives:}

At the end of the session, the health care provider will:

1. Define Asphyxia Neonatorum

2. List predisposing factors of Asphyxia Neonatorum

3. Outline the mechanism of asphyxia

4. Diagnose neonatal asphyxia

5. Assess the neonate's condition

6. Manage neonate with asphyxia

\section{TRAINING NEEDS}

\section{Knowledge Required:}

i. Predisposing factors and mechanism of Asphyxia Neonatorum

ii. Clinical features of neonatal asphyxia

iii. Describe of the APGAR score

iv. The causes of airway obstruction in a newborn baby

v. Ways of administering oxygen to a new born baby 


\section{Skills Required:}

i. Diagnosis of neonatal asphyxia

ii. Assessment of neonates condition using the APGAR score

iii. Clearing airways and stimulating the new born to cry

iv. Resuscitation of newborn

v. Give oxygen to a new born baby

vi. Counseling.

\section{Attitude Required:}

It is essential that health care providers are prepared for diagnosis of neonatal asphyxia and able to start resuscitation urgently. Good counselling skills are necessary to assist the mother to adjust to situation.

\section{SESSION 3: Sepsis in Newborn}

\section{Learning objectives:}

At the end of the session, the health care provider will:

1. Define sepsis in a newborn

2. Diagnose sepsis in the newborn

3. Manage sepsis in the neonate

4. Organize appropriate referral procedure

\section{TRAINING NEEDS}

Knowledge Required:

i. Clinical features of sepsis in the newborn

ii. Causes of sepsis in the newborn

iii. Procedure for managing cases of newborn with sepsis

\section{Skills Required:}

i. Diagnosis of sepsis in the newborn

ii. Assessment of the cause of sepsis

iii. Management of sepsis in the newborn

iv. Counselling the mother on care of the baby 


\section{Attitude Required:}

Sepsis is a major cause of neonatal mortality. Adequate and timely management is of great importance in preventing fatality. Good interpersonal skills are necessary to inform the mother and support her appropriately. Health care workers must give high priority to newborn care.

\section{Day 6: The role of health workers in abandonment of FGM/C in the context of the health, law, ethics and religion}

\section{Objectives:}

1. To provide health workers with knowledge and skills in managing complications associated with FGM/C

2. To describe the ethical implications of FGM/C

3. To describe the Laws and decrees against FGM/C and the legal implications

4. To discuss the Islam and FGM/C

\section{Knowledge:}

By the end of the weeks' training, the participants will be able to:

1. To describe the different types of FGM/C and the complications of the practice including the management of the complications

2. To discuss the rights of women and girls in different communities (specifically in the local community) and how FGM/C violates these rights

3. To discuss the legal instruments and national/international declarations relevant to the elimination of FGM/C

4. To describe the role of professional and regulatory bodies in the abandonment of FGM/C

\section{Skills:}

1. By the end of the weeks' training, the participants will be able to:

2. Manage the complications of FGM/C

3. Advocate for the rights of women, girls in their communities

4. Involve professional and regulatory bodies in FGM/C abandonment 


\section{Appendix 3: List of participants trained and facilities not represented}

\begin{tabular}{|c|c|c|}
\hline \multicolumn{3}{|c|}{ WAJIR DISTRICT } \\
\hline \multirow{6}{*}{$\begin{array}{l}\text { DHMTs Wajir } \\
\text { District }\end{array}$} & Designation & Names of DHMT \\
\hline & Nursing Officer - WDH I/c & Mohamed Hussein \\
\hline & $\mathrm{DMOH}$ & Dr. Ahmedin Omar \\
\hline & DMLT & Abdirahman Dahir Abdi \\
\hline & KECHN & Abdi Adow Aden \\
\hline & Nursing Officer - Eldas HC & Abdi Rahman Sheikh \\
\hline Type & Facility Names & Names of participants \\
\hline \multirow{13}{*}{ District Hospital } & \multirow{13}{*}{ Wajir District Hospital } & Hasan Abdub Salesa \\
\hline & & Ubah Adan Gymoi \\
\hline & & Fatuma Yusuf Ibrahim \\
\hline & & Esther Matibo \\
\hline & & Hindia Ahmed Sheikh \\
\hline & & Fatuma Adam Hussein \\
\hline & & Sahara Adow Adan \\
\hline & & Ibrahim Abdi Mohammed \\
\hline & & Mohamed N. Badel \\
\hline & & Daud Ajaba \\
\hline & & Deka M. Abdi (DPHN Office) \\
\hline & & Patrick Mite Kobha \\
\hline & & Anne Mutegi \\
\hline Health Centre & Griftu Health Centre & Cosmas Babu Ngisa \\
\hline \multirow{5}{*}{ Subdistrict Hospital } & Buna SDH & Yusuf Salat \\
\hline & & Willy Kandie \\
\hline & Bute SDH & Abdirahim Ali Adan \\
\hline & Habaswein SDH & Wesley Towett \\
\hline & Khorof Harar & 0 \\
\hline \multirow{18}{*}{ Dispensaries } & Tarbaj Dispensary & Abdullahi Bashane \\
\hline & Wagalla Dispensary & Faith Nkirote Julius \\
\hline & Albaqkorey Dispensary & 0 \\
\hline & Biyamathow Dispensary & 0 \\
\hline & Dadajabulla Dispensary & 0 \\
\hline & Dalmanyale Dispensary & 0 \\
\hline & Dambas Dispensary & 0 \\
\hline & Danaba Dispensary & 0 \\
\hline & Diff Dispensary & 0 \\
\hline & Eblen Dispensary & 0 \\
\hline & Eldas Dispensary & 0 \\
\hline & Gurar Dispensary & 0 \\
\hline & Hadado Dispensary & 0 \\
\hline & Korondille Dispensary & 0 \\
\hline & Kutulo Dispensary & 0 \\
\hline & Lagbogol Dispensary & 0 \\
\hline & Leheley Dispensary & 0 \\
\hline & Mansa Dispensary & 0 \\
\hline
\end{tabular}




\begin{tabular}{|l|l|l|}
\cline { 2 - 3 } & Riba Dispensary & 0 \\
\hline \multirow{4}{*}{} & Sabuli Dispensary & 0 \\
\cline { 2 - 3 } & Sarif Dipsensary & 0 \\
\cline { 2 - 3 } & Tulatula Dispensary & 0 \\
\cline { 2 - 3 } & Wajir-Bor Dipsensary & 0 \\
\hline
\end{tabular}

\begin{tabular}{|c|c|c|}
\hline & Designation & Names of DHMT \\
\hline \multirow{4}{*}{ DHMTs Moyale District } & DPHN & Hambule Mohamed \\
\hline & DHEO & Mohamed Kampicha \\
\hline & D Nutritionist & Sora Dima \\
\hline & Ag. DNO & Soransora \\
\hline Type & Facility Name & Names of participants \\
\hline \multirow{9}{*}{ District Hospital } & \multirow{9}{*}{ Moyale District Hospital } & Waqo Hapi Jilo \\
\hline & & Ali Marsa \\
\hline & & Rose Wato Guyo \\
\hline & & Esther Oba Kuricha \\
\hline & & Kuranja Muriungi Ntomukiri \\
\hline & & Dansoye Dulla Chudo \\
\hline & & Hassan Diba Miyo \\
\hline & & Mariam Golo \\
\hline & & Martha Tadicha \\
\hline \multirow{8}{*}{ Dispensaries } & \multirow{2}{*}{ DF Dispensary } & Shano Hussein Guyo \\
\hline & & Mokora Kimanga \\
\hline & Golole Dispensary & Harrison Juma Wendo \\
\hline & Heilu Dispensary & Mugambi G Geoffrey \\
\hline & Ramata Dispensary & Paul Guyo Waqoh \\
\hline & Uran & Moses Kibusa \\
\hline & Walda Dispensary & David Muriungi Emiliano \\
\hline & Odda Dispensary & Hawo Iman \\
\hline
\end{tabular}

\begin{tabular}{|c|c|c|}
\hline \multicolumn{3}{|c|}{ MANDERA DISTRICT } \\
\hline \multirow{6}{*}{$\begin{array}{l}\text { DHMTs Mandera } \\
\text { District }\end{array}$} & Designation & Name \\
\hline & D Pharm Tech & Isaac Maalim Eliyas \\
\hline & D Physiotherapist & Suleiman Omar \\
\hline & DPHO & A. A Mohamed \\
\hline & DHEO & Derrow Maalim Gamoh \\
\hline & NO incharge & Omondi Gogo \\
\hline Type & Facility Name & Participants' names \\
\hline \multirow{5}{*}{ District Hospital } & \multirow{5}{*}{ Mandera District Hospital } & Nurudin Abdullahi \\
\hline & & Ibrahim Isack \\
\hline & & Yunis Haji Ismail \\
\hline & & Fatuma Noor Isaac \\
\hline & & Asha Adan Farah \\
\hline \multirow{2}{*}{ Health Centers } & Banisa Health Centre & Hassan Sharif Hassan \\
\hline & Rhamu Health Centre & Omar Abdi Abdille \\
\hline
\end{tabular}




\begin{tabular}{|c|c|c|}
\hline & \multirow{6}{*}{ Takaba Health Centre } & Mohammed Ali Maalim \\
\hline & & Hassan Mohamed \\
\hline & & Abdirashid Khalif \\
\hline & & Abdinasir M Ibrahim \\
\hline & & Rukia Haji Omar \\
\hline & & Ismail Tullo Ali \\
\hline Sub district hospital & Elwak SDH & Isack Adan \\
\hline Dispensaries & Dandu Dispensary & Hassan Bulle \\
\hline & Arabia Dispensary & Abdulaziz H. Hussein \\
\hline Type & Facility Name & Participants' names \\
\hline & Kutulo Dispensary & Ali Mahat \\
\hline & Lafey Dispensary & Hassan Mohamed \\
\hline & Wargadud Dispensary & Abdi Hussein Mohamed \\
\hline & Fino Dispensary & 0 \\
\hline Dispensaries & Guba Dispensary & 0 \\
\hline & Khalalio Dispensary & 0 \\
\hline & Libehiya Dispensary & 0 \\
\hline & Rhamu- Dintu Dispensary & 0 \\
\hline & Shimbiri Fatuma Dispensary & 0 \\
\hline & Ashabito Dispensary & 0 \\
\hline & Yabicho Dispensary & 0 \\
\hline
\end{tabular}

\section{IJARA DISTRICT}

\begin{tabular}{|c|c|c|}
\hline \multirow{6}{*}{ DHMT } & Designation & Name \\
\hline & NO Maternity & Betsy Jepkorir \\
\hline & DTLC & Abdirashid Mohamed \\
\hline & $\mathrm{NO} \mathrm{MCH}$ & Rahima Yusuf Buro \\
\hline & DPHN & Siyat Moge \\
\hline & $\mathrm{CO}$ & Abdi Tawane \\
\hline \begin{tabular}{|l|} 
Type \\
\end{tabular} & Facility Name & Participants' names \\
\hline \multirow{12}{*}{ District Hospital } & \multirow{12}{*}{ Masalani District Hospital } & Suleiman Adam \\
\hline & & Mohamed Abdulahi \\
\hline & & Amos T Chelimo \\
\hline & & Fatuma Mahat \\
\hline & & Hamdi Dubat \\
\hline & & Dhahabu Hussein \\
\hline & & Rosemary Kathingati \\
\hline & & Hareth S. Dagane \\
\hline & & Douglas K Kemboi \\
\hline & & Hassan A Kassim \\
\hline & & Meimuna Ibrahim \\
\hline & & Hassan Anshur \\
\hline Health Centre & Hulugho Health Centre & Abdi Gedi Abdi \\
\hline \multirow[t]{2}{*}{ Dispensaries } & Galmagalla Dispensary & Gitonga \\
\hline & Hara Dispensary & Phorry Mwambao \\
\hline
\end{tabular}




\begin{tabular}{|l|l|l|} 
& & \\
\hline \multirow{5}{*}{ Dispensaries } & Facility Name & Participants' names \\
\cline { 2 - 3 } & Kotile Dispensary & Betty K. Nyaga \\
\cline { 2 - 3 } & \multirow{3}{*}{ Sangailu Dispensary } & Clayton Kadzenge \\
\cline { 2 - 3 } & & Wesley Kiboino \\
\cline { 2 - 3 } & Bbdullahi Hussein Ali \\
\cline { 2 - 3 } & Bothai Dipsensary & 0 \\
\hline Health Centre & ljara Health Centre & 0 \\
\hline
\end{tabular}

\section{GARISSA DISTRICT}

\begin{tabular}{|c|c|c|}
\hline \multirow{12}{*}{$\begin{array}{l}\text { DHMTs } \\
\text { Garissa } \\
\text { District }\end{array}$} & Designation & Name \\
\hline & DMLT & Hussein H.A \\
\hline & $\mathrm{CO}$ in charge $\mathrm{PGH}$ & Xaran Abdile Mohamed \\
\hline & NO & Hassan Hussein \\
\hline & $\mathrm{DMOH}$ & Dr. Warfa \\
\hline & HRIO & Martin Kamau \\
\hline & $\mathrm{CO}$ & Ahmed Abdi \\
\hline & $\mathrm{CO}$ & Abdirashid Jama \\
\hline & PNO & Mohamed Salat \\
\hline & DPHO & Ahmed A Arab \\
\hline & DPHN & Dakane Khalif \\
\hline & RH Coordinator & Mohamed Hussein Ibrahim \\
\hline Type & Facility Name & Names \\
\hline \multirow{10}{*}{$\begin{array}{l}\text { Provincial } \\
\text { General } \\
\text { Hospital }\end{array}$} & \multirow{10}{*}{ Garissa PGH } & Mohamud M. Hassan \\
\hline & & Abdirahman Kalif \\
\hline & & Nathifa Ibrahim Mohamud \\
\hline & & Ibrahim Adoh \\
\hline & & Sahara Hussein \\
\hline & & Felistus Macharia \\
\hline & & Habiba Abdulkadir \\
\hline & & Fred Ntore \\
\hline & & Beautrice Maina \\
\hline & & Tabitha Osuo \\
\hline \multirow{11}{*}{ Dispensaries } & Amuma Dispensary & Hassan Osman \\
\hline & Benane Dispensary & Omar Osman \\
\hline & Saka Dispensary & Mohamed Harun \\
\hline & \multirow[t]{2}{*}{ Medina Dispensary } & Lathan Abdi \\
\hline & & Omuga Samuel \\
\hline & Nanighi Dispensary & Wesley Kiptum \\
\hline & Police Line Dispensary & 0 \\
\hline & Prison Dispensary & 0 \\
\hline & Alinjugur Dispensary & 0 \\
\hline & Sankuri Dispensary & 0 \\
\hline & Shanta-Abaq Dispensary & 0 \\
\hline
\end{tabular}




\begin{tabular}{|c|c|c|}
\hline & Bura Dispensary & 0 \\
\hline & Danyere Dispensary & 0 \\
\hline & Dertu Dispensary & 0 \\
\hline & Dujis Dispensary & 0 \\
\hline & GTTC Dispensary & 0 \\
\hline & Gurufa Dispensary & 0 \\
\hline & Korakora Dispensary & 0 \\
\hline & Mansabubu Dispensary & 0 \\
\hline & Kulan & 0 \\
\hline & \multirow{5}{*}{ GTZ/UNHCR Dadaab Refugee Camp } & Mohamed Bashir Dahir \\
\hline & & Fatuma Kuno \\
\hline & & Ahmed Wardere \\
\hline & & Halima Dahir \\
\hline & & Artha Mohamed \\
\hline \multirow{6}{*}{ Dispensaies } & \multirow{6}{*}{ SIMAHO } & Rumano Noor \\
\hline & & Lydia Semedi Karkia \\
\hline & & Cecilia M. Mwanzia \\
\hline & & Fatuma Ibrahim \\
\hline & & Zahra Hussein \\
\hline & & Rukia Hassan \\
\hline
\end{tabular}

\section{Appendix 4 Task assignments for participants at Garissa PGH}

\section{$\underline{\mathrm{VCT} / \mathrm{Lab} \text { Team Visit }}$}

1. Look at the environment where the VCT / Lab is situated / cleanliness / appearance / convenience.

2. Patient flow \& general arrangement of department.

3. Over view of services provided (From I/c)

4. The process (steps\} in VCT; - who does what? Number of trained officers on VCT - Number on site (duty\}, sequence of event (steps\}.

5. Records kept - registers used/correct entry method applied, number seen, and confidentiality, how staff relate to one another.

6. Counseling techniques applied - pre/post test \& follow ups - follow the process.

7. Specific test done - its specificity and sensitivity.

8. ARV's given, types, criteria used to know who is to get $A R V^{\prime}$ 's, reagents available Vs requirement, other non VCT related tests done.

9. Health Education / teaching aids available- H/E talks, posters, pamphlets, video shows, models, availability of condoms - male / female. 
10. Infection prevention techniques practiced- what do they disinfect? What do they use? Is it effective in your opinion? who does it? For how long? Types of protective gears provided and are they used? Staff knowledge on IP, how many were trained? Do they have IPC and are active?

11. Time taken - from the time client comes to the facility until he or she leaves it.

12. Identify types of hazardous waste generated / observed at VCT / Lab and how they are disposed?

13. Networking between facility staff and community members for HIV/AIDS patients- home based care (HBC).

14. Constraints faced by staff/ short falls and remedial measures planned by the staff to overcome the same.

\section{Maternity/Labour Ward Rotation:}

\section{Group 1}

1. Basic equipment and supplies needed in the labor ward e.g consumables, like gloves, cotton wool, gauze, syringes and needles etc. Check availability and accessibility

2. Review the records required in the maternity ward such as: cardex, registers, patient notes, drug registers, DBR, duty roster, MDR etc. Review how they should be kept and how they should be updated and stored

3. Admission process: If possible admit one client to the labor ward

4. Review handing over of patient at the end of the shift. Practice the process of handing over

5. Define the duties of a nurse / RCO in the labor ward and maternity. Importance of the nursing care plan

6. Basic drugs needed in the maternity and labor ward unit, e.g ergometrine, antibiotics, syntocinon, magnesium sulphate, diazepam, etc. Check accessibility and availability

7. Basic tests done in the maternity / labor ward e.g $\mathrm{Hb}$, blood grouping and $\mathrm{x}-$ matching, etc. Are request forms and specimen bottles available? Can they interpret results and institute interventions

8. General patient care e.g uniform, bedding, nets cleanliness, etc 
Group 2

1. Partograph: Review thoroughly the use of the partograph

2. Demonstrate different case scenarios on the partograph eg obstructed labor, fetal distress, prolonged labor, in coordinate uterine contractions, and when and how to intervene

3. Review old case notes especially the partograph

4. Monitoring of the patient antepartum,and post partum; e.g vital signs, breasts, lochia loss, uterine involution, episiotomy

5. Review of patients admitted to the ward: antepartum, post C/S, post partum; clerk and discuss management

6. Observe/perform de-infibulation if indicated

\section{Group 3}

1. Review infection prevention and waste disposal practices

2. Check the emergency tray and discuss emergency preparedness

3. Manage a woman in labour and conduct delivery if patient available

\section{MCH/FP Clinic Team}

Major problems

1. Anaemia

2. Malaria in pregnancy

3. HIV/AIDs

4. Blood pressure during pregnancy

Emphasize

1. Haematinics for all pregnant women

2. Prophylaxis (sp) for all pregnant women

3. Correct anaemia during pregnancy

4. Emergency preparedness and individualized birth plan

5. Routine checking and recording of urine proteins and blood pressure. 
1. Environment - check for:
a. Cleanliness
b. Flow of patients
c. General arrangement of facility including chairs, tables, coaches
d. Health education before the other services
e. Adequacy of space
f. Privacy

2. Registration area/room/weighing area - check:
a) Registers/cards/reports
b) Adequacy and functioning of equipments
c) Staffing

3. ANC Room - check:
a) Staffing
b) IPCC
c) Record systems - registers, ANC cards, monitoring charts etc
d) Patient/clients care - IPCC, IP, health education e.g IBP
e) Drugs available for routine care - preventive
f) Emergency tray
g) Availability of water
h) Infection prevention
i) Client attendance - daily and monthly
j) Waste disposal

FP Room - check for the following;
a) Organization of the room
b) Staffing
c) Type of the FP method available
d) Attend to clients
e) Infection prevention: running water, system used to sterilize the equipment
f) No of patients attendance per day/month
g) Recording/storage of information
h) IPCC 


\section{PMTCT Room}

- Organization of the room - flow of the clients

- Staffing; Number and qualification

- Test used

- Utilization: No of client seen daily/monthly; No. positive; No. negative

- Recording/storage of information

- Adequate space; Privacy/confidentially

- Waste disposal

- Storage of reagent

- Counseling - how is done, individually or group

- Adequate supply of supplies 